\title{
The glucose-sensing transcription factor MLX promotes myogenesis via myokine signaling
}

\author{
Liam C. Hunt, ${ }^{1}$ Beisi Xu, ${ }^{2}$ David Finkelstein, ${ }^{2}$ Yiping Fan, ${ }^{2}$ Patrick A. Carroll, ${ }^{3}$ Pei-Feng Cheng, ${ }^{3}$ \\ Robert N. Eisenman, ${ }^{3}$ and Fabio Demontis ${ }^{1}$ \\ ${ }^{1}$ Department of Developmental Neurobiology, Division of Developmental Biology, St. Jude Children's Research Hospital, \\ Memphis, Tennessee 38105, USA; ${ }^{2}$ Department of Computational Biology, St. Jude Children's Research Hospital, Memphis, \\ Tennessee 38105, USA; ${ }^{3}$ Division of Basic Sciences, Fred Hutchinson Cancer Research Center, Seattle, Washington 98109, USA
}

\begin{abstract}
Metabolic stress and changes in nutrient levels modulate many aspects of skeletal muscle function during aging and disease. Growth factors and cytokines secreted by skeletal muscle, known as myokines, are important signaling factors, but it is largely unknown whether they modulate muscle growth and differentiation in response to nutrients. Here, we found that changes in glucose levels increase the activity of the glucose-responsive transcription factor MLX (Max-like protein X), which promotes and is necessary for myoblast fusion. MLX promotes myogenesis not via an adjustment of glucose metabolism but rather by inducing the expression of several myokines, including insulinlike growth factor 2 (IGF2), whereas RNAi and dominant-negative MLX reduce IGF2 expression and block myogenesis. This phenotype is rescued by conditioned medium from control muscle cells and by recombinant IGF2, which activates the myogenic kinase Akt. Importantly, MLX-null mice display decreased IGF2 induction and diminished muscle regeneration in response to injury, indicating that the myogenic function of MLX is manifested in vivo. Thus, glucose is a signaling molecule that regulates myogenesis and muscle regeneration via MLX/IGF2/Akt signaling.
\end{abstract}

[Keywords: MLX; IGF2; myokine; glucose; myogenesis; regeneration; skeletal muscle]

Supplemental material is available for this article.

Received July 6, 2015; revised version accepted October 26, 2015.

Unicellular and multicellular organisms have evolved several homeostatic adaptive strategies to regulate cell and organismal behavior in the face of fluctuating nutrient levels. Skeletal muscle is one of the tissues that are most profoundly remodeled in response to changes in the levels of glucose and other nutrients. Many aspects of skeletal muscle homeostasis are modulated by glucose, including myogenesis, the process by which mononucleated progenitors (myoblasts) fuse into syncytial muscle cells during development and regeneration. Several lines of evidence indeed point to glucose as an important mediator of myogenesis. First, high glucose levels promote myogenesis (Nedachi et al. 2008), and the expression of genes involved in glucose metabolism is up-regulated during myogenesis in vitro (Webster et al. 1990). Second, glucose metabolism via glycolysis is necessary for myogenesis in the Drosophila embryo (Tixier et al. 2013). Last, glucose restriction inhibits myogenesis by activating AMPK kinase and the sirtuin SIRT1, and partial loss of SIRT1 allows myogenesis to

Corresponding author: fabio.demontis@stjude.org Article published online ahead of print. Article and publication date are online at http://www.genesdev.org/cgi/doi/10.1101/gad.267419.115. occur even at low glucose concentrations that are normally not permissive (Fulco et al. 2008). Despite these studies, the mechanisms and signaling pathways sensing glucose and responsible for glucose-induced adaptive myogenesis are largely unknown.

A class of proteins that have recently gained attention for their many signaling roles is myokines; i.e., growth factors and cytokines secreted by skeletal muscle in response to a range of different stimuli (Pedersen and Febbraio 2012; Demontis et al. 2013a). Myokines can act locally on the muscle itself as well as systemically on other tissues to regulate many physiological functions ranging from metabolic homeostasis to aging and disease-related processes (Boström et al. 2012; Demontis et al. 2014). In addition to endocrine functions, there are many examples of autocrine and paracrine signaling of myokines on the muscle, including the modulation of myogenesis (Rai and Demontis 2015). For example, leukemia inhibitory factor (LIF) is a

(C) 2015 Hunt et al. This article is distributed exclusively by Cold Spring Harbor Laboratory Press for the first six months after the full-issue publication date (see http://genesdev.cshlp.org/site/misc/terms.xhtml). After six months, it is available under a Creative Commons License (Attribution-NonCommercial 4.0 International), as described at http:// creativecommons.org/licenses/by-nc/4.0/. 
myokine that regulates myoblast proliferation and differentiation and is produced in response to exercise (Hunt et al. 2010, 2011; Broholm et al. 2011). However, despite the important signaling roles of myokines, little is known about the transcription factors that modulate their expression in response to glucose and other nutrients.

Max-like protein X (MLX) is a member of the Myc-Max family of basic helix-loop-helix (bHLH) transcription factors (Billin et al. 1999) that, in response to high glucose levels, translocates from the cytoplasm to the nucleus (Peterson et al. 2010), where it regulates the expression of target genes with carbohydrate response element (CHORE) motifs in their promoter (Minn et al. 2005). MLX is ubiquitously expressed across tissues and regulates transcription by forming heterodimers with either MLX-interacting protein (MLXIP/MondoA) or MLXIPlike (MLXIPL/CHREBP) that are primarily expressed in the muscle (Billin et al. 2000) and liver (Yamashita et al. 2001), respectively. Increased intracellular glucose leads to nuclear accumulation of MLX (along with MLXIP and MLXIPL) and increased DNA binding. Several lines of evidence support the hypothesis that glucose-6-phosphate is the primary metabolite responsible for MLX activation. First, MLX translocation to the nucleus and transcriptional activity can be stimulated by 2-deoxyglucose, an analog of glucose that accumulates in cells following phosphorylation but cannot be further metabolized (Stoltzman et al. 2008). Second, phosphorylation of glucose by hexokinase is required for MLX activity (Stoltzman et al. 2008). Last, phosphoglucoisomerase deficiency leads to glucose-6-phosphate buildup and enhances MLX activation (Stoltzman et al. 2011). The precise mechanisms by which glucose-6-phosphate activates MLX are not completely understood but perhaps entail direct binding to MLXIP/ MLXIPL (McFerrin and Atchley 2012). In addition, glucose-dependent post-translational modifications can regulate MLXIPL (Guinez et al. 2011), which can also be phosphorylated and inactivated by the energy-sensing kinase AMPK (Kawaguchi et al. 2002). Thus, MLX and its coactivators can sense glucose via multiple mechanisms. Furthermore, because MLX is part of a large interactive network of transcriptions factors, including Myc, Max, Mxd, and MLXIP/MLXIPL, its transcriptional output may be determined by the context-specific organization of this network (Diolaiti et al. 2015).

In agreement with a fundamental role for MLX in glucose sensing, microarray studies indicate that $\sim 75 \%$ of all transcriptional changes induced by glucose in hepatocytes are mediated by MLX (Ma et al. 2006). For example, MLX induces the expression of enzymes involved in carbon metabolism (Ma et al. 2005, 2006; lizuka et al. 2009) and proteins that regulate glucose uptake, including thioredoxin-interacting protein (TXNIP) (Parikh et al. 2007; $\mathrm{Wu}$ et al. 2013). However, although MLX is important for glucose sensing and glucose metabolism in the liver (Postic et al. 2007), it is unknown whether it has similar roles in other tissues.

In this study, we examined the transcriptional and metabolic roles of MLX in skeletal muscle cells. We found that MLX transcriptional activity increases in response to glucose and that MLX is necessary for and promotes myogenic differentiation. However, rather than promoting glucose metabolism, MLX induces the expression of many myokines in response to glucose, including insulin-like growth factor 2 (IGF2), which recapitulates the effects of MLX on myogenic differentiation. These findings indicate that MLX modulates glucose-induced myoblast fusion and differentiation via myokine signaling.

\section{Results}

The transcription factor $M L X$ induces target gene expression in muscle cells via glucose-induced DNA binding and histone $\mathrm{H} 4$ acetylation

To experimentally modulate MLX transcriptional activity in muscle cells, wild-type MLX (MLX-WT), dominantnegative MLX (MLX-DN), MLX RNAi (MLX shRNA), and N-terminally truncated MLX (MLX- $\mathrm{N} 121)$ constructs were generated and stably expressed in $\mathrm{C} 2 \mathrm{C} 12$ mouse myoblasts via lentiviral-mediated transduction. Overexpression of MLX variants led to an 60-fold increase in $M l x$ mRNA levels compared with empty vector control, whereas MLX shRNA led to an $80 \%$ decrease in endogenous Mlx mRNA levels compared with a nontargeting control shRNA (Fig. 1A; Supplemental Fig. S1A). MLX-WT, MLX-DN, and MLX- $\triangle$ N121 protein variants were detected by Western blot at the expected sizes ( 20 kDa for MLX- $\triangle$ N121 and $\sim 30 \mathrm{kDa}$ for MLX-WT and MLX-DN) (Supplemental Fig. S1B) and by immunostaining of lentiviral-transduced cells that coexpress GFP (Supplemental Fig. S1C). Both MLX-WT and MLX-DN were ubiquitously detected in the cells, whereas MLX$\Delta$ N121 localized to nuclei (Supplemental Fig. S1C), consistent with previous evidence that the N-terminal domain of MLX harbors cytoplasmic retention sequences (Billin et al. 2000).

To assess the transcriptional activity of transgenic MLX variants, we examined changes in the expression of Txnip and Arrdc4, two genes known to be induced by glucose and MLX (Stoltzman et al. 2008; Yu et al. 2009). Both MLX-DN and MLX shRNA prevented glucose-induced expression of Txnip and Arrdc4, whereas MLX-WT significantly enhanced their expression (Fig. 1B). Moreover MLX- $\Delta$ N121 blunted Txnip and Arrdc4 induction by glucose, indicating that $\mathrm{N}$-terminal truncation impairs MLX transcriptional activity, although it leads to constitutively nuclear localization (Supplemental Fig. S1A-C).

To further corroborate these findings, we assessed the DNA-binding capacities of MLX variants to the CHOREcontaining Txnip promoter by chromatin immunoprecipitation (ChIP) combined with quantitative PCR (qPCR). Expression of MLX-WT allowed for glucose-dependent recruitment of MLX and MLXIP (MondoA) to the Txnip promoter, whereas this was blocked by MLX-DN but retained with MLX- $\Delta$ N121 (Supplemental Fig. S1D). Furthermore, histone $\mathrm{H} 4$ acetylation, an epigenetic mark induced by glucose and indicative of open chromatin and active transcription, was increased at the Txnip promoter in response to glucose and MLX-WT but inhibited by 
A
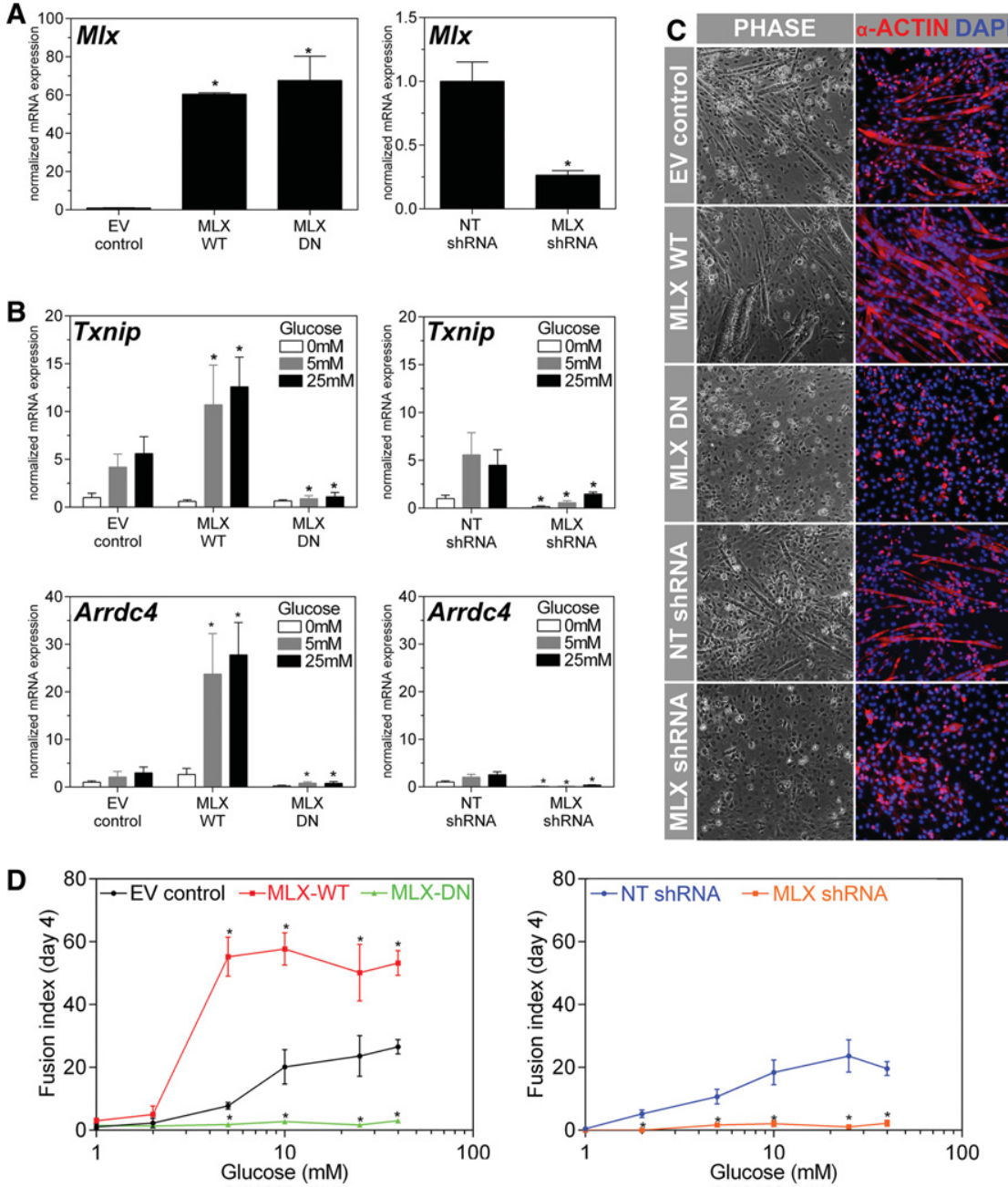

Figure 1. The transcription factor MLX regulates glucose-induced myogenesis. (A) Increased $M 7 x$ expression is detected by quantitative PCR (qPCR) in muscle cells stably expressing MLX-WT and MLX-DN compared with control cells expressing an empty vector (EV) control. MLX shRNA decreases Mlx levels compared with a nontargeting (NT) control shRNA. (B) MLX protein variants and MLX shRNA regulate the expression of MLX target genes known to be glucose-responsive. MLX-WT increases the expression of Txnip and Arrdc4, which are also induced by glucose. Conversely, MLX-DN and MLX shRNA decrease Txnip and Arrdc4 expression and prevent their glucose-induced upregulation. The histograms show the mean of three biological replicates \pm SEM. $\left(^{*}\right) P<$ 0.05. See also Supplemental Figure S1. $(C)$ Phase contrast and $a$-actin immunostaining demonstrate increased myotube formation at day 4 of differentiation due to MLX-WT overexpression and impairment of myogenesis due to loss of MLX activity (MLX-DN overexpression and MLX shRNA) compared with controls (empty vector [EV] and a nontargeting [NT] shRNA). See also Supplemental Figures S2 and S3. (D) The fusion index (i.e., the number of nuclei within syncytial myotubes) indicates that MLX-WT increases myoblast fusion, whereas MLX-DN and shRNA decrease myoblast fusion. Myoblasts stably expressing MLX-WT have increased glucose sensitivity and increased fusion index at lower glucose concentrations that are normally not permissive. Conversely, myoblasts stably expressing MLX-DN and MLX shRNA cannot fuse regardless of glucose concentrations. $\left.{ }^{*}\right) P<0.05$, SEM. $n=3$.
MLX-DN and MLX- $\Delta$ N121 (Supplemental Fig. S1D). Therefore, nuclear localization of MLX is insufficient for transcriptional activity and requires recruitment of additional transcriptional coactivators, such as histone acetyl transferases, as previously described (Davies et al. 2008; Peterson et al. 2010). Taken together, these findings indicate that the transcription factor MLX modulates glucose-induced transcriptional responses in muscle cells and that both its $\mathrm{N}$-terminal domain and DNA binding are required for histone $\mathrm{H} 4$ acetylation to activate target gene expression in muscle cells.

Regulation of MLX target genes in muscle cells may also stem from the interaction of MLX with other bHLH transcription factors at target gene promoters. In particular, MLX has recently been shown to coordinately regulate a subset of target genes with c-Myc (Shen et al. 2015) and to be necessary for survival of Myc-driven tumors (Carroll et al. 2015). We found that c-Myc occupancy at the Txnip promoter is reciprocal to MLX and likely mediated by E-boxes contained within the CHORE motifs (Supplemental Fig. S1D). Therefore, antagonistic promoter occupancy by MLX and c-Myc may also contribute to determining the transcriptional output of MLX target genes in muscle cells.

\section{$M L X$ regulates myogenesis in response to glucose}

C2C12 myoblasts form syncytial muscle cells (myotubes) when grown to confluence in cell culture medium containing 10\% fetal bovine serum (FBS) and subsequently cultured for $8 \mathrm{~d}$ in differentiation-inducing medium containing $2 \%$ horse serum. As measured by immunostaining (Fig. 1C), control myoblasts differentiated efficiently and reached maximal fusion by $4 \mathrm{~d}$ of differentiation, whereas MLX-DN and MLX shRNA myoblasts appeared unable to fuse. Conversely, MLX-WT myoblasts fused more rapidly than controls and produced hypertrophic myotubes, as indicated by the fusion index, which is the proportion of nuclei present within myotubes (Supplemental Fig. S2A). These findings indicate that MLX activity is necessary for and promotes myogenesis. To determine whether myotube hypertrophy caused by MLX-WT is simply due to enhanced myoblast fusion or is also due to additional anabolic effects, we next analyzed the proportional 
myotube size, which is defined as the area of the myotubes divided by the number of myotube nuclei (Supplemental Fig. S2A). Interestingly, the proportional myotube size did not significantly change in response to MLX-WT overexpression, indicating that myotube hypertrophy is primarily due to the higher number of myoblasts fusing to form myotubes.

Because MLX transcriptional activity is regulated by glucose, we next probed their interaction by examining the extent of myoblast fusion at day 4 of differentiation in response to different levels of MLX activity and glucose. Myoblast fusion was maximal at 10-25 mM glucose concentrations in control cells (Fig. 1D), whereas myoblasts overexpressing MLX-WT appeared more glucose-sensitive and had maximal fusion at relatively low glucose concentrations $(5 \mathrm{mM})$. Conversely, myoblasts overexpressing MLX-DN or MLX shRNA were glucose-insensitive and unable to fuse even at high glucose concentrations (40 mM). These findings indicate that MLX and glucose are indeed required for myoblast fusion and that MLX activity dictates cellular sensitivity to glucose.

To dissect the mechanisms involved, we tested whether MLX modulates cell cycle exit, myogenic commitment (nuclear detection of myogenin), and expression of genes encoding proteins that mediate myoblast fusion. However, none of these myogenic processes were modulated by MLX (Supplemental Fig. S2A,B). Moreover, restoring normal levels of Txnip, a key MLX target gene, did not rescue the fusion defects of MLX-DN myoblasts (Supplemental Fig. S3A,B). Because previous studies have shown that glucose uptake and metabolism promote myogenesis (Fulco et al. 2008), we next tested whether MLX induces these processes in muscle cells as it does in the liver (Ma et al. 2005; Postic et al. 2007; Stoltzman et al. 2008; Peterson et al. 2010). Surprisingly, glucose uptake was unperturbed in myoblasts overexpressing MLX-WT (Fig. 2A) and even increased in MLX-DN and MLX shRNA myoblasts com- pared with controls, indicating that intracellular glucose levels are not limiting for myogenesis in myoblasts with loss of MLX function (Fig. 2A). Moreover, the levels of lactate (an end product of glycolysis) (Fig. 2B) and the extracellular acidification rate (ECAR; a measure of glycolytic activity) (Fig. 2C) were not modulated by MLX activity, nor was the oxygen consumption rate (OCR), an indicator of mitochondrial respiration (Fig. 2D). Thus, in contrast to its function in the liver, MLX does not regulate glucose metabolism in myoblasts, suggesting that signaling rather than metabolic adaptive responses is induced by MLX to regulate myogenesis.

\section{$M L X$ regulates myokine expression}

In order to discover novel genes regulated by MLX that could be responsible for its effects on myogenesis, we performed RNA sequencing (RNA-seq) of cells with MLX gain and loss of function (Supplemental Table S1). RNAseq analyses found many genes significantly regulated in muscle cells overexpressing MLX-WT, MLX-DN, and MLX shRNA compared with their respective controls (Fig. 3A). To identify MLX target genes relevant for myogenesis, we focused on a set of 80 genes that were significantly and concordantly regulated by MLX-WT, MLXDN, and MLX shRNA (see Materials and Methods for selection criteria). Of these, 62 genes were transcriptionally activated by MLX, whereas 18 genes were repressed. Enriched gene clusters were identified by DAVID (Database for Annotation Visualization and Integrated Discovery) and PIR (Protein Information Resource) annotation keywords and indicated that there was a significant enrichment (31 out of 67 annotated genes) for muscle-secreted proteins (i.e., myokines) (Fig. 3B), indicating an unanticipated role of MLX in regulating their expression.

Considering that genes encoding muscle-secreted signaling proteins (myokines) constitute a major class of
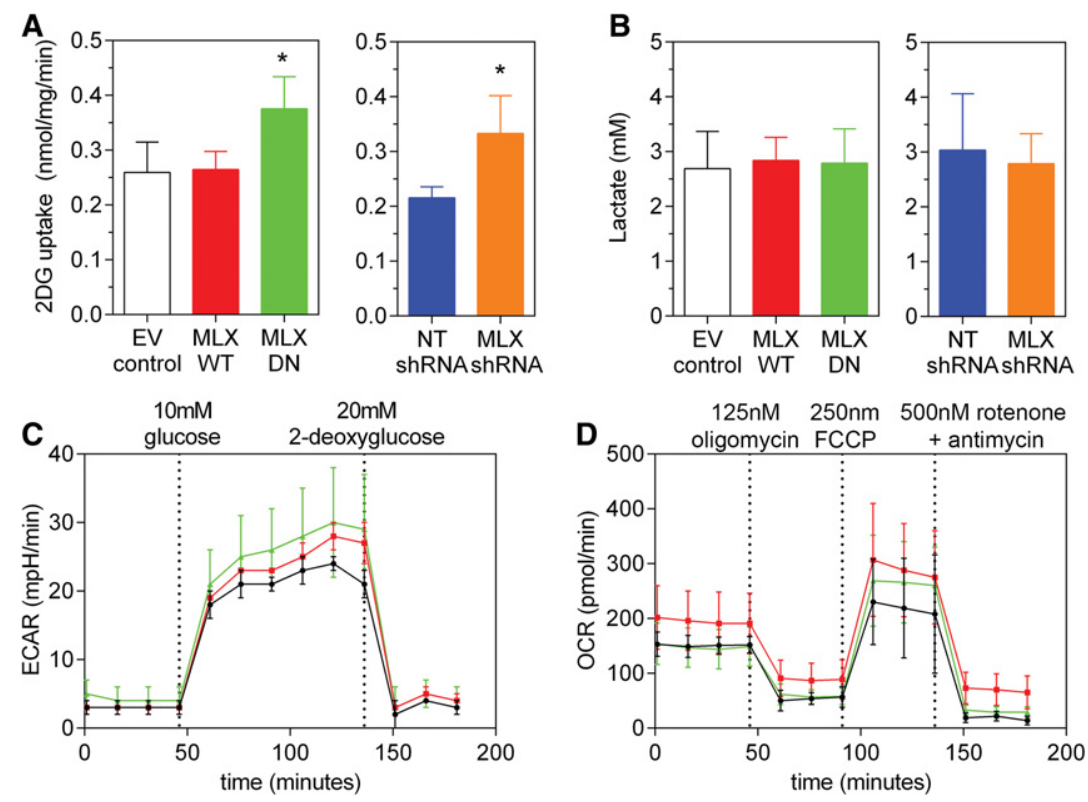

Figure 2. The promyogenic effects of MLX are not due to changes in glucose metabolism. $(A)$ 2-deoxy-D-glucose (2DG) uptake does not change with MLX-WT overexpression but increases with MLX loss of function (MLX-DN and MLX shRNA). $\left({ }^{*}\right) P<0.05$, SEM. $n=3$. $(B)$ Levels of extracellular lactate, an end product of glucose metabolism, are not affected by MLX activity. $(C)$ Similarly, the ECAR, which measures glycolytic conversion of pyruvate into lactate, is not altered by changes in MLX activity. (D) The OCR, indicative of mitochondrial respiratory capacity, is also unchanged. (Red) MLX-WT; (green) MLX-DN; (black) empty vector (EV) control. 


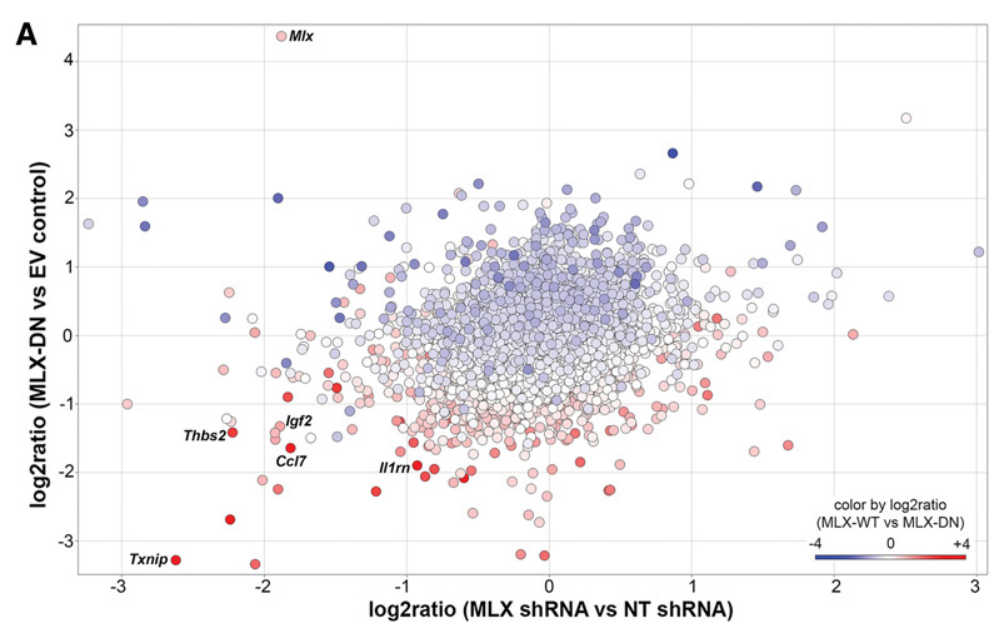

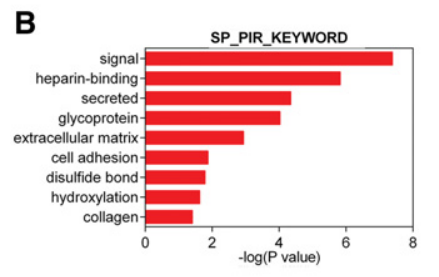

D

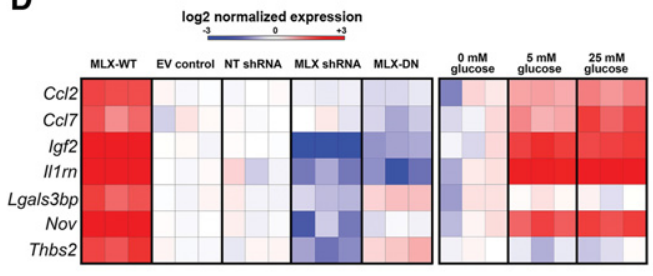

C control

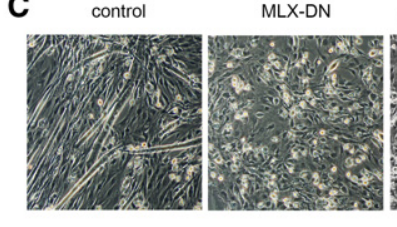

E

E $N T$ shRnA $M L X$ shRnA

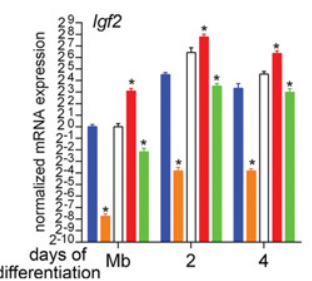

Figure 3. MLX regulates the expression of several myokines. (A) Plot of RNA-seq data comparing $\log _{2}$ fold changes in gene expression observed in cells overexpressing MLX-DN ( $Y$-axis) versus $\log _{2}$ fold changes seen in MLX shRNA cells ( $X$-axis). Points are colorcoded according to the $\log _{2}$ fold changes in gene expression seen in cells overexpressing MLX-WT. Many of the genes transcriptionally regulated by MLX encode for myokines; i.e., muscle-secreted proteins (31 out of 67 MLX-regulated annotated genes). See also Supplemental Table S1. (B) Enriched gene set analysis shows that MLX regulates predominantly the expression of extracellular and secreted proteins. (C) Fusion defects observed in myoblasts overexpressing MLX-DN are rescued by conditioned culture medium from control myoblasts. $(D)$ Normalized expression of qPCR data indicates that MLX-WT and glucose strongly induce the expression of several myokines, whereas MLX shRNA and MLX-DN reduce their expression. Data from three biological replicates are shown. (E) Igf2 expression increases during myogenesis, suggesting that it is important for myoblast fusion and differentiation. Moreover, Igf2 expression is increased by MLX-WT and decreased by loss of MLX activity (MLX-DN and MLX shRNA). The histograms show the mean of three biological replicates \pm SEM. $\left(^{*}\right) P<0.05$. See also Supplemental Figure S4.
MLX-regulated genes, we tested whether conditioned media from differentiating control cells can rescue the fusion defects of myoblasts overexpressing MLX-DN. Interestingly, the addition of this milieu of secreted factors stimulated fusion of MLX-DN myoblasts, resulting in the formation of myotubes after 4 din culture (Fig. 3C), suggesting that myokines may be required for glucose-induced myogenesis.

From the RNA-seq data of MLX-regulated myokines, we selected the chemokines Ccl2 and Ccl7, the insulin-like growth factor $I g f 2$, the interleukin-1 receptor antagonist II1rn, the lectin-binding protein $L g a l s 3 b p$, the matricellular CCN family gene Nov, and the thrombospondin Thbs2 and examined them further because of their potential association with myogenesis (Florini et al. 1991a; Iruela-Arispe et al. 1993; Sakamoto et al. 2002; Yahiaoui et al. 2008). First, by qPCR, we validated their expression in response to MLX activity and found that MLX-WT increases their mRNA levels, whereas MLX-DN and MLX shRNA decrease their expression relative to controls (Fig. 3D). Second, we tested whether MLX-induced myokines are modulated during differentiation and by changes in glucose levels. The expression of Cc12, Cc17, Igf2, I11rn, and Nov increased in response to higher glucose levels (Fig. 3D), whereas the expression of most myokines did not increase following differentiation (Supplemental Fig. S4A), with the exception of Igf2 expression, which increased (Fig. 3E). These findings indicate that glucose and MLX regulate the expression of myokines produced by myoblasts.

\section{MLX promotes myokine gene transcription by increasing histone $\mathrm{H} 4$ acetylation}

Glucose influences gene expression by inducing chromatin remodeling via epigenetic modifications such as acetylation of histones H3 and H4 (Lee et al. 2014). Considering that MLX increases $\mathrm{H} 4$ acetylation in proximity to Txnip (Supplemental Fig. S1D; Cha-Molstad et al. 2009), we performed ChIP of acetylated histone H4 (H4ac) from MLX-WT-expressing and MLX-DN-expressing cells followed by sequencing (Fig. 4) to determine whether MLX generally regulates $\mathrm{H} 4$ acetylation in proximity to its target genes. Myoblasts with MLX-DN showed overall increased $\mathrm{H} 4 \mathrm{ac}$ near all gene promoters (Fig. 4A), which could be explained by increased glucose uptake by these cells (Fig. 2A). However, MLX-WT myoblasts displayed increased H4ac specifically in the promoter regions of genes up-regulated by MLX-WT (as determined from RNA-seq data) (Fig. 3) and decreased $\mathrm{H} 4 \mathrm{ac}$ in the promoter regions of down-regulated genes (Fig. 4A; Supplemental Table S2). Interestingly, there 
A

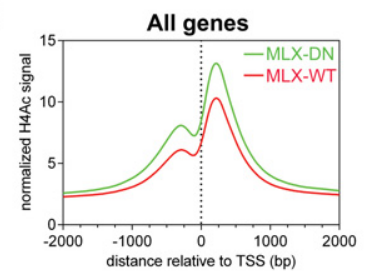

B Arrdc4

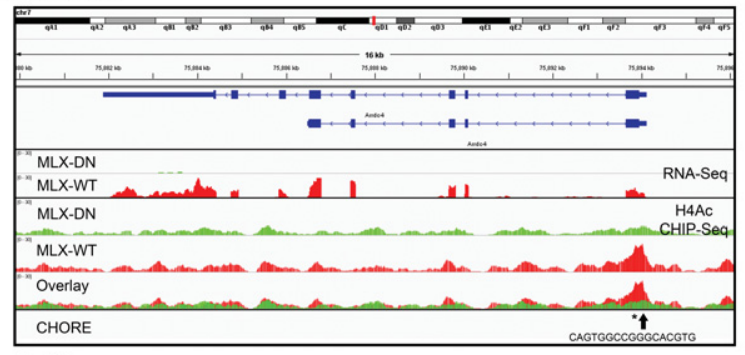

lgf2

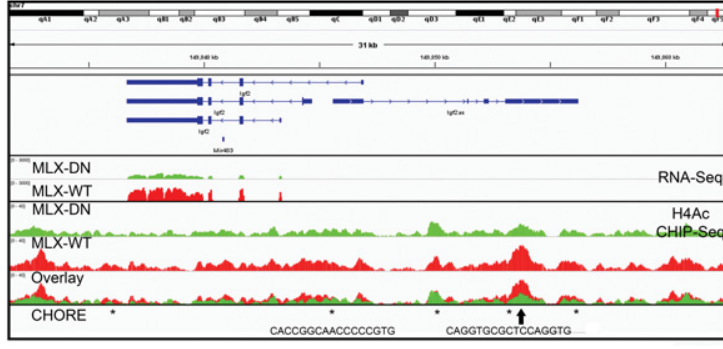

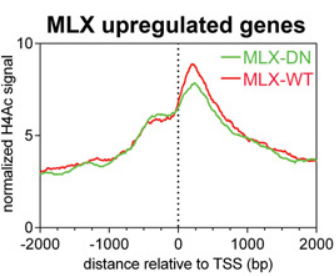
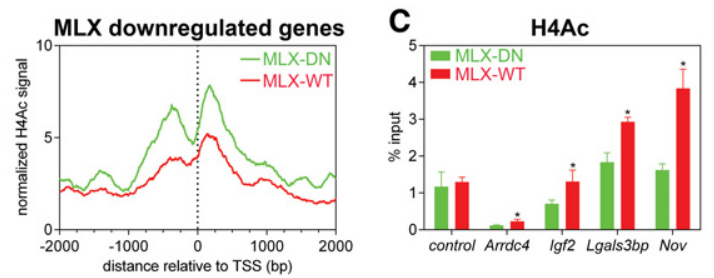

Lgals3bp

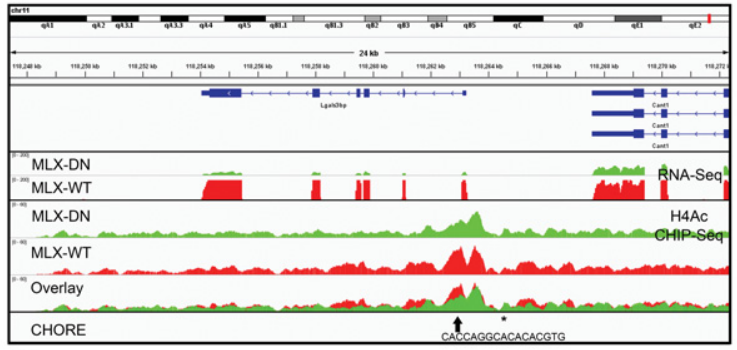

Nov

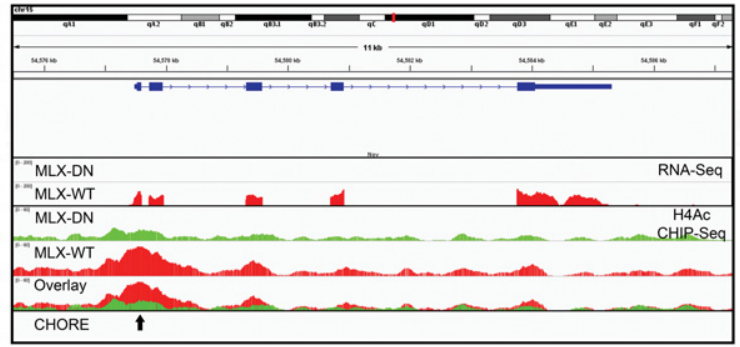

Figure 4. Histone $\mathrm{H} 4$ acetylation occurs specifically at MLX target genes and is dependent on MLX DNA binding. (A) ChIP-seq (ChIP combined with deep sequencing) data for H4ac demonstrate overall H4ac enrichment around the TSSs of genes in MLX-WT and MLXDN myoblasts and the presence of nucleosome-free regions in close proximity to the TSSs. Overall, a global increase in H4ac levels is observed in MLX-DN cells compared with MLX-WT. However, genes specifically up-regulated by MLX-WT /and having decreased expression with MLX-DN and MLX shRNA) show increased H4 acetylation, whereas genes down-regulated by MLX show decreased H4 acetylation. (B) Examples of MLX target genes that show increased H4ac levels in MLX-WT compared with MLX-DN. RNA-seq tracks show transcript levels, and computationally identified MLX-binding CHOREs (denoted with an asterisk) that lie in close proximity to the H4ac peaks are identified below. (C) ChIP-qPCR validation of H4ac enrichment at the promoter regions of MLX target genes in response to overexpression of MLX-WT (red) compared with MLX-DN (green). Arrows in B indicate regions displaying H4ac peaks that have been further validated by ChIP-qPCR. See also Supplemental Figure S5 and Supplemental Tables S2 and S3.

was an overall correlation between the relative levels of H4ac and the presence of predicted MLX-binding sites; $\mathrm{i}$. e., CHORE-like motifs (Fig. 4B; Supplemental Table S3). For example, the glucose- and MLX-regulated gene Arrdc4 showed a strong H4ac signal in MLX-WT but not in MLXDN cells surrounding the transcriptional start site (TSS), which also contains a CHORE motif. Similarly, the myokine Lgals3bp displayed strong H4ac levels at the $+1 \mathrm{nu}-$ cleosome position in MLX-WT cells, and this was absent in MLX-DN cells. Also in this case, there was a CHORE upstream of the TSS. This indicates that MLX activity is needed for the up-regulation of $\mathrm{H} 4$ acetylation locally at promoter regions of MLX target genes, including myokines, presumably via its binding to CHORE motifs. We also validated these changes in $\mathrm{H} 4 \mathrm{ac}$ by qPCR and found similar results (Fig. 4C). Moreover, several other MLX target genes are similarly regulated (Supplemental Fig. S5; Supplemental Tables S2, S3).

Although the association between CHOREs and H4ac may hold true for several genes, CHOREs could not be identified near Nov, which had strong MLX-dependent H4ac centered on its TSS, suggesting possible diversions from this model. Moreover, although there were several CHOREs and H4ac peaks present within the Igf2 gene locus, the $\mathrm{H} 4 \mathrm{ac}$ peak with the greatest magnitude of change caused by MLX was present in the final exon of Igf2as (Igf2 antisense) (Fig. 4B, arrow). This peak also had three CHORE motifs in its vicinity (two of which contained the canonical sequence CAGGTGnnnnnCAGGTG). Although this is at somewhat of a distance $(<10 \mathrm{~kb})$ from the TSS, there are well-known long-range enhancers and promoters that drive Igf2 transcription and can be as far as $100 \mathrm{~kb}$ away from the TSS (Alzhanov et al. 2010). Furthermore, the site of this MLX-dependent H4ac peak has already been described as an Igf2 promoter (dubbed the $\mathrm{Pm}$ promoter) that can drive transcription of Igf2 in myoblasts (Duputié et al. 2012). On this basis, we propose that DNA binding of MLX occurs via CHORE-like sequences and that it promotes $\mathrm{H} 4$ acetylation near target genes, including Igf2 and other myokines. 
IGF2 is a myokine regulated by $M L X$ and promotes myogenesis

We tested whether exogenous addition of any of these MLX-induced myokines regulates myoblast fusion. Recombinant mouse myokines were added to differentiation media of control and MLX-DN-overexpressing myoblasts at concentrations of 1,10 , and $100 \mathrm{ng} / \mathrm{mL}$, and myotube formation was monitored. No recombinant myokine was able by itself to induce fusion of MLX-DN myoblasts (Supplemental Fig. S4B), except for 10 and $100 \mathrm{ng} / \mathrm{mL}$ IGF2 (Fig. 5A). Concentrations of 100 ng/mL IGF2 also enhanced fusion of control myoblasts (Fig. 5A).

Given the capacity of IGF2 to rescue the myoblast fusion defects due to MLX-DN, we tested whether Igf2 transcriptional changes induced by MLX indeed result in corresponding changes in IGF2 protein levels. To this purpose, we measured IGF2 protein levels by ELISA from undifferentiated myoblasts and differentiating cultures at days 2 and 4. Measurement of IGF2 levels in conditioned medium and cell lysates indicated that IGF2 protein levels increase during myogenesis and in response to MLX-WT overexpression, particularly in the conditioned medium but also in the cell lysates. Conversely, IGF2 protein levels were low in myoblasts overexpressing MLX-DN and MLX shRNA (Fig. 5B). This confirms that changes in Igf2 expression lead to corresponding changes in intracellular and, most notably, secreted IGF2 protein levels.
To test whether changes in IGF2 protein levels induce corresponding changes in IGF2 signaling, we monitored by Western blot phospho-Akt (Ser473) levels, which increase in response to exogenous IGF2. As predicted by the progressive increase in Igf2 expression and IGF2 protein levels during myogenesis, phosphorylation of Akt increases during the course of muscle differentiation. Moreover, increased Akt phosphorylation was found in response to MLX-WT overexpression, whereas myoblasts with MLX-DN and MLX shRNA had lower phospho-Akt levels (Fig. 5C), consistent with IGF2 mRNA and protein changes elicited by MLX gain and loss of function. IGF2 has been previously shown to be produced by myoblasts (Florini et al. 1991a; Erbay et al. 2003) and stimulate myogenic differentiation via Akt (Florini et al. 1991b; Yoon and Chen 2008; Markljung et al. 2009). To test whether Akt is indeed a key mediator of myoblast fusion downstream from MLX/IGF2 signaling, we used pharmacological inhibitors of Akt and found that they prevented MLXand IGF2-induced myoblast fusion following induction of differentiation (Supplemental Fig. S6A-C).

We then tested the responsiveness of MLX-WT-overexpressing and MLX-DN-overexpressing cells to IGF2. In control cells, concentrations of IGF2 between 50 and $100 \mathrm{ng} / \mathrm{mL}$ increased the fusion index from control levels of $25 \%$ up to $40 \%$. When exposed to exogenous IGF2, myoblasts overexpressing MLX-DN gradually increased their fusion index from $0 \%$ (with $1 \mathrm{ng} / \mathrm{mL}$ IGF2) up to

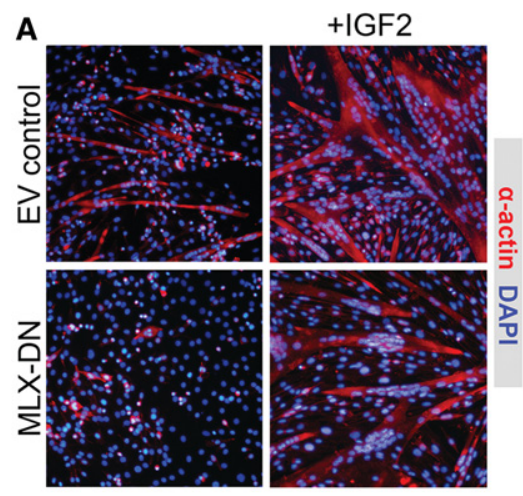

D

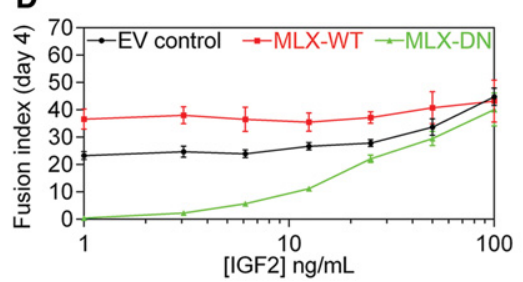

B

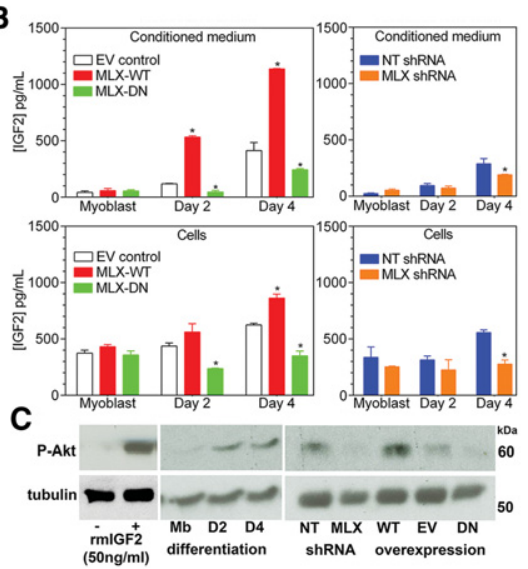

E

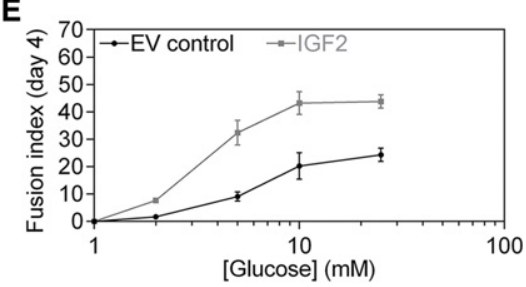

Figure 5. MLX promotes myogenesis via IGF2 signaling. (A) Of several MLX-induced myokines, only IGF2 induces myogenesis and rescues myoblast fusion defects caused by MLX-DN overexpression. Immunostaining for $a$-actin (red) and DAPI (4',6-diamidino-2phenylindole) (blue) is shown. See also Supplemental Figure S4. (B) ELISA measurement of IGF2 levels in conditioned cell culture medium and cellular lysates indicates that IGF2 protein levels increase as differentiation proceeds and in response to MLX activity (MLX-WT), whereas loss of MLX function (MLX-DN and MLX shRNA) decreases IGF2 protein levels. The histograms show the mean of three different experiments \pm SEM. $\left(^{*}\right) P<0.05$. $(C)$ Treatment of serum-deprived muscle cells (-) with recombinant mouse IGF2 $(+)$ increases phospho-Akt levels (Ser473). Akt phosphorylation also increases during myogenesis ([Mb] myoblast; [D2] day 2 of differentiation; [D4] day 4 of differentiation) coincident with increased Igf2 mRNA and protein levels. Loss of MLX function (MLX-DN and MLX shRNA) decreases Akt phosphorylation, whereas MLX-WT increases it, indicating that MLX regulates IGF2/Akt signaling. (D) The dose response curve of the myoblast fusion index in response to IGF2 levels indicates that while exogenous addition of recombinant mouse IGF2 to culture medium stimulates fusion in control cells, myoblasts overexpressing MLX-WT are insensitive and appear to have already reached a maximum level of myoblast fusion. In addition, IGF-2 rescues myoblast fusion defects due to MLX-DN overexpression. (E) Myoblasts overexpressing IGF2 have increased fusion at low levels of glucose that are normally not permissive for fusion, similar to MLX-WT overexpression (shown in $D$ ). The mean of three biological replicates \pm SEM is shown, with $P<0.05$ compared with the respective control at the same concentration of IGF2/glucose. 
normal control levels (25\%) with $25 \mathrm{ng} / \mathrm{mL}$ IGF2 and further increased to $40 \%$ at the maximum IGF2 concentration of $100 \mathrm{ng} / \mathrm{mL}$. The fusion index of myoblasts overexpressing MLX-WT remained steady at $\sim 40 \%$ with all concentration of IGF2 ranging from 1 to $100 \mathrm{ng} / \mathrm{mL}$, suggesting that myoblast fusion is already maximal in these cells due to MLX-induced Igf2 expression (Fig. 5D). Similarly, myoblasts overexpressing IGF2 displayed a higher fusion index than control cells at all glucose concentrations, including low glucose levels that are typically not permissive for myoblast fusion (Fig. 5E). Therefore, there is strong interdependence between MLX, IGF2, and glucose levels during myogenesis. Altogether, our data are consistent with an important role for autocrine and paracrine IGF2/Akt signaling in stimulating myoblast fusion and differentiation downstream from MLX.

\section{Muscle regeneration is impaired in muscles of MLX-null mice}

To further investigate the role of MLX in myogenesis, we analyzed the muscles of MLX-null mice $\left(\mathrm{MlX}^{-1-}\right)$. The tibialis anterior muscles from 12 -wk-old mice lacking MLX appeared essentially normal when compared with wild-type $\left(\mathrm{MlX}^{+/+}\right)$controls, with no signs of pathology or impaired muscle development (Fig. 6A), suggesting that fetal myogenesis is unaffected by loss of MLX. Measurement of muscle and myofiber size revealed that, indeed, there were no changes in the proportional area of tibialis anterior muscles from control and $\mathrm{Mlx}^{-/-}$mice, although there was a slight increase in the proportion of larger myofibers in $\mathrm{Mlx}^{-1-}$ muscles (Fig. 6B). In response to injury, myogenesis can also occur in adults in order to regenerate damaged muscles. Importantly, IGF2 is a key myokine known to regulate adult muscle regeneration (Ge et al. 2009; Ikemoto-Uezumi et al. 2015). We therefore tested whether MLX regulates muscle regeneration via IGF2 signaling. When injected with cardiotoxin, tibialis anterior muscles of wild-type mice efficiently removed necrotic myofibers and displayed regenerating centrally nucleated myotubes $7 \mathrm{~d}$ after injury, whereas MLX-null mice had persistent necrotic myofibers and reduced fusion of myotubes, indicating impaired regeneration (Fig. 6A). Remarkably, cardiotoxin-injured tibialis anterior muscles from $\mathrm{MlX}^{-/-}$mice displayed impaired regeneration exemplified by an overall decrease in muscle area accompanied by an increased proportion of smaller-sized myotubes compared with isogenic controls (Fig. 6B). Consistent with reduced myoblast fusion due to the absence of MLX, the expression of its transcriptional targets was also perturbed in regenerating muscle. Decreased $M 7 x$ mRNA levels coincided with a severe loss of Txnip expression, which was not influenced by cardiotoxin (Fig. 6C). Igf2 mRNA, on the other hand, was dramatically up-regulated following cardiotoxin injury, but the increase in Igf2 expression was significantly blunted in $\mathrm{Mlx}^{-/-}$muscles, similar to what is seen in myoblasts lacking MLX activity (Figs. $3,4)$. Other myokines regulated by MLX that were identified from RNA-seq (Fig. 3), including Thbs2, Colba1, Col6a2, and Nov, showed transcriptional regulation simi- lar to that of Igf2 (Supplemental Fig. S7), suggesting that they may also contribute to muscle regeneration. Altogether, these findings indicate that MLX is necessary for efficient muscle regeneration in vivo and that this is due to its capacity to regulate the expression of Igf2 and other myokines.

\section{Discussion}

Insulin resistance and diminished intracellular uptake of glucose are components of both aging and diabetes, but it is largely unknown whether they are causally associated with the decline in the regeneration of muscle and other tissues that is observed in these conditions. One possibility is that limited glucose uptake leads to lower ATP production and thus indirectly compromises regeneration by affecting cellular resources available for this process. Alternatively, glucose may act as a signaling molecule that directly modulates pathways responsible for regeneration. This study demonstrates a previously unanticipated role for the glucose-sensing transcription factor MLX in regulating the differentiation and growth of muscle cells via myokine signaling (Fig. 7), suggesting that altered MLX signaling may contribute to decreased regeneration in many disease conditions.

Much of the research on MLX to date has focused on its role in sensing changes in glucose levels in hepatocytes and concordantly adjusting glucose metabolism by increasing the expression of target genes involved in glycolysis and negatively regulating glucose uptake (Ma et al. 2005; Postic et al. 2007; Stoltzman et al. 2008; Peterson et al. 2010). However, we found that MLX does not regulate glycolysis in muscle precursor cells. Moreover, although MLX increases the expression of Txnip (a negative regulator of glucose uptake) in muscle cells, we observed no changes in the expression of other reported MLX target genes associated with glucose metabolism. Therefore, glucose metabolism does not account for the modulation of myogenesis by MLX gain and loss of function. However, muscle progenitor cells have low glucose uptake and metabolism compared with contracting muscle cells, suggesting that MLX may regulate glucose metabolism in other circumstances. In line with this model, muscle-specific loss of MLXIP does not affect glucose metabolism in the resting state but leads to higher lactate levels in response to short-term fasting and exercise (Minako et al. 2014). This paradoxical effect, opposite to what is expected, is due to the interaction of MLXIP with PGC$1 \alpha$ and does not require CHORE motifs and MLX (Minako et al. 2014). Future studies will determine whether MLX can influence glucose metabolism in muscles under specific circumstances.

Surprisingly, we found that MLX regulates the expression of several muscle-secreted signaling proteins (myokines) that are also induced by glucose. Among these, IGF2 recapitulated the effects of MLX in modulating glucose-induced myogenesis by activating the kinase Akt, which is known to be required for myoblast fusion downstream from IGF2 (Jiang et al. 1999). In glucose-restricted 
A
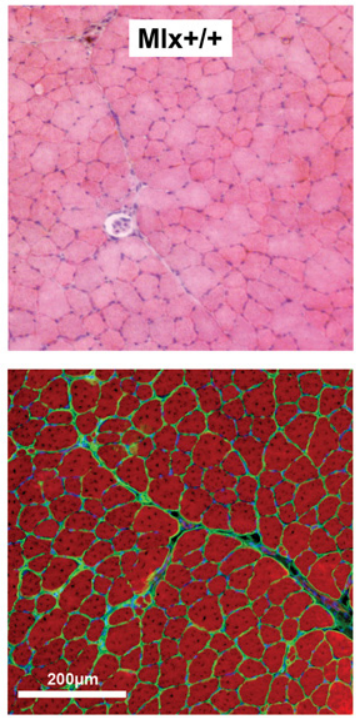

\section{B}

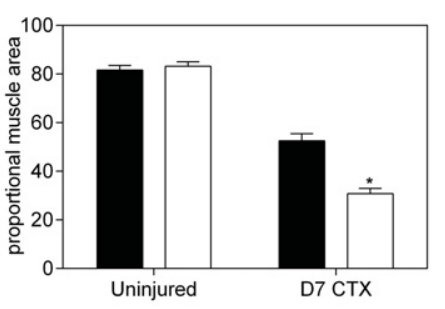

C

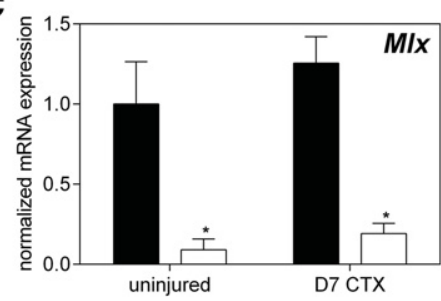

uninjured
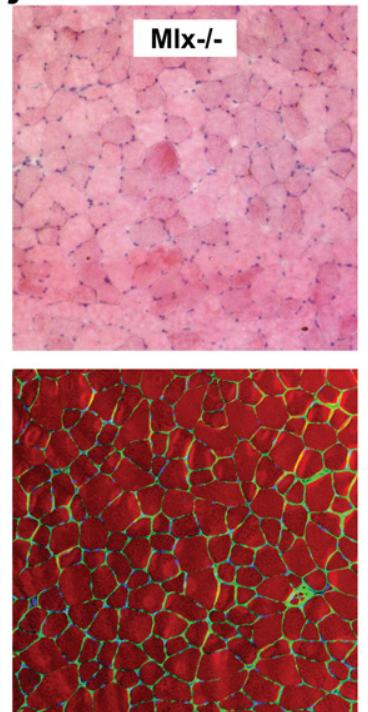

D7 CTX
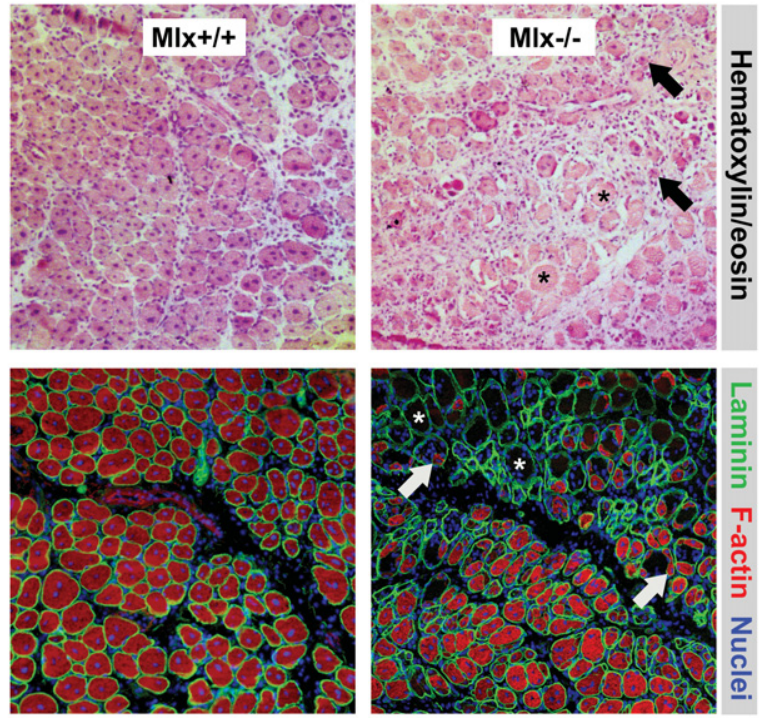
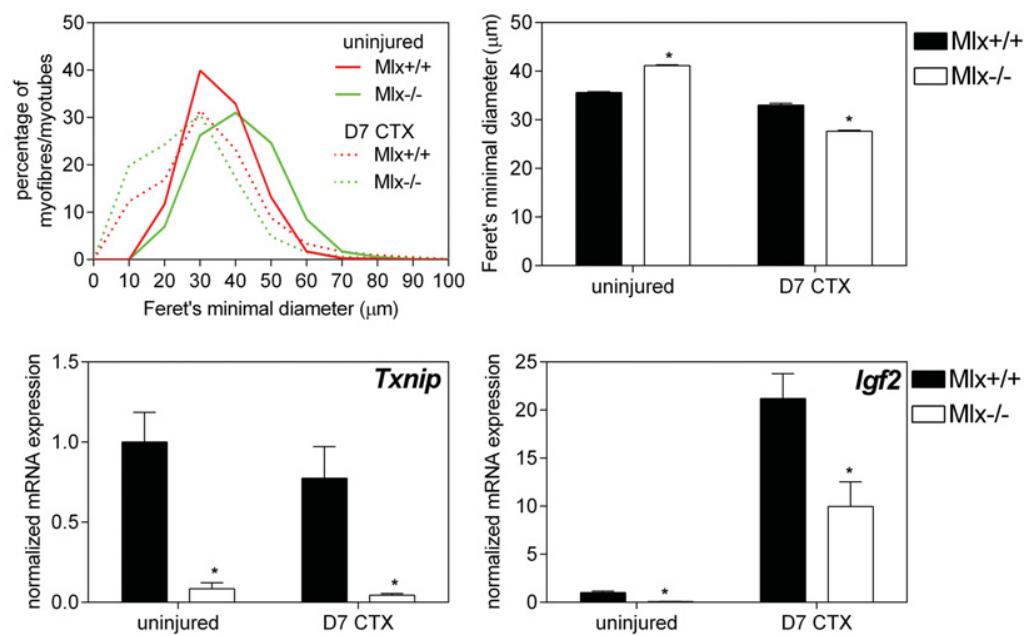

Figure 6. Mlx-null mice have impaired muscle regeneration. (A) Representative histology images from uninjured and injured tibialis anterior muscles undergoing regeneration $7 \mathrm{~d}$ after cardiotoxin injection (D7 CTX) from Mlx-null $\left(\mathrm{Mlx}^{-/-}\right)$and wild-type $\left(M 7 \mathrm{x}^{+/+}\right)$isogenic control mice at $12 \mathrm{wk}$ of age. The top panels show hematoxylin/eosin staining, whereas the bottom panels show immunostaining for Laminin (green; a marker defining the external myofiber boundaries), nuclei (blue), and F-actin (red; which indicates viable myofibers, whereas its absence defines necrotic myofibers). In wild-type mice, muscle regeneration is completed $7 \mathrm{~d}$ after cardiotoxin injection (D7 CTX), resulting in the formation of myofibers completely filling external boundaries. Conversely, Mlx-null mice have impaired muscle regeneration, as indicated by persistence of numerous necrotic myofibers (indicated by asterisks) and the presence of small myotubes (indicated by arrows) that incompletely fill external boundaries left by prior myofibers. $(B)$ The proportional muscle area (calculated as the percentage of total muscle area occupied by F-actin-positive myofibers) is similar in Mlx-null and wild-type muscles in uninjured conditions but is decreased significantly in regenerating $M l x$-null muscles compared with wild-type controls. This indicates that muscle regeneration is impaired due to insufficient myogenesis in Mlx-null mice. Frequency distributions of myofiber and myotube diameters show a small shift to the right in uninjured Mlx-null myofibers compared with wild-type. However, there is an increased proportion of small myotubes (<30 $\mu \mathrm{m}$ in diameter) $7 \mathrm{~d}$ after cardiotoxin injection in $M l x$-null muscles compared with wild-type controls, indicating impaired regeneration and insufficient fusion of Mlx-null myoblasts. Coincident changes are seen in the mean diameter of myotubes. Frequency distributions represent $>2000$ cell measurements pooled from individual animals for each genotype and condition. $(C)$ qPCR analysis of $M l x$ and Txnip levels confirms that MLX activity is decreased in muscles from Mlx-null mice. Moreover, Igf2 up-regulation induced by cardiotoxin injection is significantly blunted in Mlx-null muscles, which explains their defective regeneration. The histograms show the mean of four or more biological replicates \pm SEM. $\left(^{*}\right) P<0.05$ compared with the wild-type isogenic controls.

conditions, myoblasts with increased MLX activity and IGF2 levels indeed fused to a much greater degree than control myoblasts, indicating that glucose-induced signal- ing mediated by MLX/IGF2/Akt can promote myogenesis in unfavorable conditions. These findings are in line with a previous study that has shown that glucose can promote 


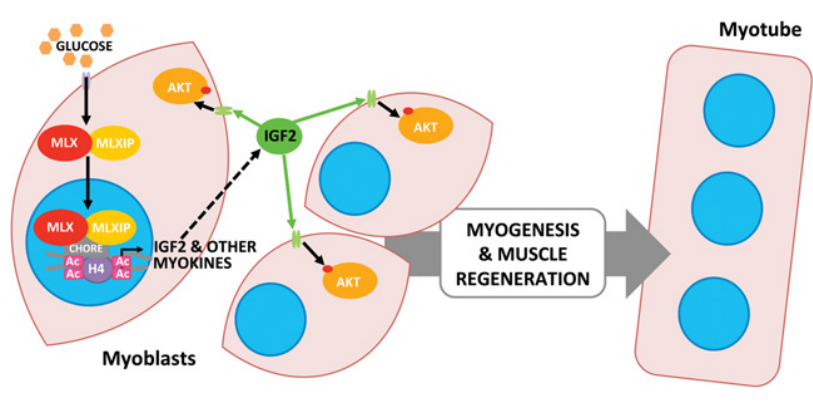

Figure 7. The glucose-sensing transcription factor MLX promotes myogenesis via myokine signaling. Diagram that summarizes the role of MLX in myogenesis and muscle regeneration. In response to glucose, MLX is activated in myoblasts, translocates from the cytoplasm to the nucleus, binds to DNA CHORE motifs, increases histone $\mathrm{H} 4$ acetylation (Ac) at target gene promoters, and increases the expression of target genes. Myokines such as IGF2 are transcriptionally activated by MLX. Once secreted, IGF2 can in turn activate cell-autonomously and cell-nonautonomously the myogenic kinase Akt by promoting its phosphorylation. Akt promotes myoblast fusion and differentiation and thus allows for formation of syncytial muscle cells (myotubes). We propose that the decreased muscle regenerative capacity observed during aging and diseases characterized by altered glucose homeostasis may be due to altered MLX and myokine signaling.

myogenesis by decreasing the activity of the energy-sensing AMPK kinase (Fulco et al. 2008). In this respect, it is interesting to note that AMPK can also inhibit nuclear translocation and transcriptional activity of MLX heterodimers via phosphorylation (Kawaguchi et al. 2002), suggesting that low glucose can block myogenesis by inhibiting MLX/IGF2 signaling via AMPK. Moreover, AMPK inhibits mTOR, with which both MLX (Carroll et al. 2015) and MLXIP (Kaadige et al. 2015) functionally interact, suggesting multiple levels of cross-talk between MLX and nutrient-sensing pathways. We therefore propose that the glucose-sensing transcription factor MLX and myokine signaling may encompass a novel part of the molecular pathway necessary for glucose-induced adaptive myogenesis. Given its widespread expression, MLX may modulate the transcription of secreted signaling proteins in response to glucose in many cell types, which was also implicated by a recent study in colorectal cancer cells (Nam et al. 2015). Therefore, our study indicates that MLX has more diverse roles than previously anticipated, presumably in concert with the large network of positive and negative transcriptional regulatory factors to which it belongs (Diolaiti et al. 2015).

We discovered a role for MLX in the production of IGF2 and other myokines by myoblasts in response to glucose. Although Igf2 expression was known to increase during muscle differentiation (Kou and Rotwein 1993) and regeneration (Levinovitz et al. 1992) and to modulate these processes, there have not been any transcriptional activators of Igf2 described to date, and only negative regulators of Igf2 expression were known (Markljung et al. 2009; Duputié et al. 2012). We found that glucose and MLX induce Igf2 transcription, whereas MLX-DN and MLX shRNA reduce its expression. Our study therefore ad- dresses this important area of research by demonstrating that MLX transcriptionally activates Igf2 expression in a glucose-dependent manner during myogenesis. Although the presence of MLX-binding sites (CHORE motifs) and modulation of histone $\mathrm{H} 4$ acetylation suggest a direct role for MLX in regulating Igf2 expression, it remains possible that indirect mechanisms are also involved.

Consistent with our findings that MLX regulates myoblast fusion and differentiation in vitro, we found that muscle regeneration is impaired in Mlx-null mice. Compared with controls, loss of MLX resulted in persistence of necrotic myofibers, reduced myotube fusion, lower proportional muscle area, and decreased expression of Igf2 and other injury-induced myokines. Both the reduced myotube fusion and the persistence of necrotic myofibers observed in Mlx-null muscles undergoing regeneration could be explained by reduced Igf2 expression because regenerating aged muscles (which have reduced Igf2 expression) of wild-type mice display similar alterations, and these can be rescued by IGF2 administration (IkemotoUezumi et al. 2015). Therefore, IGF2 is a key mediator of the myogenic functions of MLX. However, MLX induces the expression of several other myokines in addition to IGF2, and therefore it is possible that regeneration deficits observed in muscles of Mlx-null mice derive also from reduced transcription of these myokines.

The remarkable similarity between the impaired regeneration of aged wild-type mice and young $M 7 x$-null mice suggests a possible role for MLX in the maintenance of muscle homeostasis throughout aging via regulation of myokine expression. On this basis, MLX could delay the progression of sarcopenia (the age-related loss of skeletal muscle mass and function) and promote muscle growth and regeneration in the context of many debilitative diseases characterized by muscle atrophy (Demontis et al. 2013b; Piccirillo et al. 2014).

In summary, we found that MLX is a transcriptional sensor of glucose levels in muscle cells and that it orchestrates adaptive glucose-induced myogenesis by regulating the expression of several myokines. IGF2 is a key MLX-induced myokine that stimulates myoblast fusion and differentiation via Akt in response to glucose and muscle injury. Considering that the activity of glucose-responsive pathways changes during development, aging, and diseases characterized by metabolic dyshomeostasis, our findings provide a possible mechanistic basis for understanding how glucose acts as a signaling molecule to control muscle cell fate in different physiologic and pathologic contexts.

\section{Materials and methods}

\section{Cell culture methods}

The mouse myoblast C2C12 cell line (American Type Culture Collection, CRL-1772) was maintained in standard conditions; i.e., in high-glucose (4.5 g/L, equivalent to $25 \mathrm{mM})$ DMEM containing 10\% FBS and supplemented with GlutaMax (Life Technologies) in a $5 \% \mathrm{CO}_{2}$ incubator at $37^{\circ} \mathrm{C}$. To induce differentiation, $\mathrm{C} 2 \mathrm{C} 12$ cells were grown to near confluence in $10 \%$ FBS-containing growth medium and subsequently in 
differentiation medium containing $2 \%$ normal horse serum in DMEM, which was changed daily. Quantitation of the fusion index (i.e., the proportion of total nuclei present within syncytial myotubes with three or more nuclei) was calculated following 8 $\mathrm{d}$ of differentiation using confluent myoblasts (at day 0 of differentiation, immediately prior to the addition of differentiation medi$\mathrm{um}$ ) as a comparison. Because a plateau in myoblast fusion was observed by day 4 of differentiation, most experiments were done at day 4 of differentiation. For rescue experiments with conditioned cell culture medium, myoblasts overexpressing MLX$\mathrm{DN}$ were cultured with conditioned culture medium from differentiating entry vector control myoblasts, and the medium was replaced every day for $4 \mathrm{~d}$ before imaging by phase contrast microscopy. For experiments with recombinant myokines, these were added to the differentiation cell culture medium at $100 \mathrm{ng} /$ $\mathrm{mL}$, and the medium was replaced daily until day 4 before immunostaining. Myotube formation with $100 \mathrm{ng} / \mathrm{mL}$ recombinant protein is shown in Figure 4C and Supplemental Figure S4. Recombinant myokines were purchased from the following suppliers: NOV from Genetex (GTX48170-PRO), IL1RN from Abnova (P4498), LGALS3BP from Abnova (H00003959-P01), IGF2 from Biolegend (588204), and CCL2 and CCL7 from RND Biosystems (479-JE-010/CF and 456-MC-010). The HEK293 subcloned cell line $293 \mathrm{FT}$, which was used to produce lentiviral vectors, was maintained in $10 \%$ FBS containing DMEM.

\section{Skeletal muscle regeneration experiments in mice}

All mice were housed and handled in accordance with approved St. Jude Children's Research Hospital Institutional Animal Care and Use Committee protocols. C57BL6 mice carrying MLX-null mutations were generated by partial deletion of the Mlx coding sequence and are described in detail by PA Carroll and RN Eisenman (in prep.). Mice were genotyped before experimental use, and lack of MLX activity was confirmed by qPCR for Txnip and other MLX target genes. For skeletal muscle regeneration experiments, $50 \mu \mathrm{L}$ of cardiotoxin was injected into the tibialis anterior muscle of 12wk-old male mice at a concentration of $0.1 \mathrm{mg} / \mathrm{mL}$ in PBS. Seven days after injection, the muscles were excised and frozen in isopentane cooled with liquid nitrogen for histology and snap-frozen in liquid nitrogen for RNA extractions and qRT-PCR. Frozen sections were cut to $10 \mu \mathrm{m}$ and fixed for $15 \mathrm{~min}$ with $4 \%$ paraformaldehyde. Sections were then washed, blocked, and immunostained for Laminin (clone 4H8-2; Santa Cruz Biotechnology, sc-59854), DAPI (4',6-diamidino-2-phenylindole), and Alexa fluor 633-conjugated phallodin and analyzed with the Nikon NIS Elements software to determine the Feret's minimal diameter of myofibers and myotubes using Laminin and phalloidin staining, respectively.

\section{Cloning}

To obtain a vector for overexpression of MLX-WT, its cDNA (NM_01550.3) was cloned by PCR into the MCS of the pCDH-EF1-MCS-T2A-GFP lentiviral expression vector (System Biosciences) in-frame with T2A-GFP and sequence-verified. MLX- $\Delta$ N121 (a putative constitutive active) (Iizuka et al. 2009) was obtained by PCR and consisted of nucleotides 360-894 of the MLX ORF. A MLX-DN construct was obtained by mutagenesis of MLX-WT by introducing the R141A and R142S mutations, which have been previously shown to allow for heterodimerization but prevent DNA binding, thus inhibiting transcription (Ma et al. 2005). Control nontargeting and MLX targeting lentiviral shRNAs were purchased from Origene (TL513600) in a pGFPC-shLentiviral vector.

\section{Lentiviral transduction}

Lentiviral-mediated transduction of myoblasts was achieved by cotransfecting 293FT cells with the lentiviral long-terminal repeat-containing expression vectors alongside the packaging vectors pCMV8.9 and pVSV-G to produce VSV-G pseudo-typed lentiviral vectors. Viral titers were measured by serial dilution and transduction of 293FT cells. C2C12 cells were then transduced with lentiviral vectors at a multiplicity of infection of 100 with $8 \mu \mathrm{g} / \mathrm{mL}$ polybrene for $24 \mathrm{~h}$ and expanded. pCDH-EF1MCS-T2A-GFP lentiviral transduced cells were 100\% GFP-positive and maintained transgenic GFP and MLX expression persistently. Following transduction, shRNA transduced cells were further selected with $2 \mu \mathrm{g} / \mathrm{mL}$ puromycin for $7 \mathrm{~d}$, and knockdown was tested by qPCR. TG513600A/GI580306 MLX shRNA resulted in a $>70 \%$ decrease in MLX mRNA levels relative to the nontargeting shRNA (TR30021) control (Fig. 1), while the other three shRNA were not efficacious in knocking down MLX levels (data not shown).

\section{ELISA}

For measuring IGF2 levels in conditioned cell culture medium and cell lysates, the mouse IGF2 Duoset ELISA kit (RND Biosystems, DY792) was used following the manufacturer's instructions. Conditioned medium was collected after $8 \mathrm{~h}$ of incubation with cells, whereas cell lysates were obtained by washing cells with PBS with protease inhibitors and lysing them in PBS by freezing-thawing three times. The conditioned medium and lysate samples were centrifuged, and the supernatants were used for measuring IGF2 levels.

\section{Western blot}

Four days after differentiation, muscle cells cultured in 12-well plates were washed with cold PBS and lysed on ice with $200 \mu \mathrm{L}$ of RIPA buffer with protease and phosphatase inhibitors. Protein concentrations were quantitated with the Bio-Rad protein assay, and all samples were standardized in order to load $20 \mu$ of protein per polyacrylamide gel lane. PAGE was performed, and proteins were transferred to PVDF membranes for probing. Membranes were blocked with $5 \%$ milk powder and incubated with primary antibodies overnight at $4^{\circ} \mathrm{C}$ before incubation with secondary antibodies for $2 \mathrm{~h}$ and chemiluminescence imaging. Primary antibodies used were rabbit anti-phospho-Akt (Ser473) D9E (Cell Signaling, 4060), rabbit anti-phospho-Erk antibody (Cell Signaling, 9101), rabbit anti a-tubulin 11H10 (Cell Signaling, 2125), and mouse polyclonal anti-MLX (Abcam, ab167286; this weak-affinity antibody detects MLX upon overexpression but not its endogenous levels).

\section{Immunostaining}

Cells were fixed by adding 1 vol of $4 \%$ paraformaldehyde to 1 vol of culture medium and incubating for $15 \mathrm{~min}$ at room temperature. Cells were then permeabilized with $0.1 \%$ Triton X-100, blocked with $2 \%$ BSA and $2 \%$ horse serum for $2 \mathrm{~h}$, and then incubated with primary antibody overnight at $4{ }^{\circ} \mathrm{C}$ followed by washes and incubation with Alexa fluor 488- and 555-conjugated secondary antibodies and DAPI. The primary antibodies used were mouse polyclonal anti-MLX (Abcam, ab167286), mouse IgM anti-a-actin 5C5 (Santa Cruz Biotechnology, sc-58670), and mouse anti-myogenin F5D (Dako, IR067), all at a dilution of 1:100. Myogenin levels were assessed by immunostaining after differentiation because myoblasts are myogenin-negative prior to addition of differentiation medium. EDU staining was 
performed following EDU labeling for $2 \mathrm{~h}$ in culture and following the recommended instructions using the Click-iT EdU Alexa fluor 555 imaging kit (Life Technologies, C10338).

\section{Image analysis}

Image analysis was done with ImageJ (Schneider et al. 2012). ImageJ was used to derive the fusion index by counting the nuclei present in syncytial myotubes and within mononucleated myoblasts, the percentage of total nuclei within myotubes, and the percentage of EDU- and myogenin-positive nuclei. ImageJ was also used to quantitate the area of myotubes to derive the myotube size to nuclei ratio.

\section{ChIP}

ChIP was performed from $\mathrm{C} 2 \mathrm{C} 12$ cells cultured in $15-\mathrm{cm}$ dishes following fixation in $1 \%$ formaldehyde for $10 \mathrm{~min}$, quenching with $125 \mathrm{mM}$ glycine for $5 \mathrm{~min}$, washing with PBS, and collection of the cells by centrifugation. Subsequently, Active Motif's ChIPIT Express kit (catalog no. 53008) was used following the manufacturer's instructions. In brief, collected cells were lysed with kit buffers and dounce-homogenized. The nuclear pellet was then sonicated, and chromatin was sheared using a Diagenode Bioruptor with 10 pulses each of 17 sec on and 43 sec off. Chromatin shearing efficiency was assessed by sample purification and gel electrophoresis and consistently yielded sheared chromatin within the range of 200-1000 base pairs (bp), with intense foci around $300-400 \mathrm{bp}$. Once chromatin shearing was confirmed, immunoprecipitation was performed by incubating $3 \mu \mathrm{g}$ of antibody with $40 \mu \mathrm{L}$ of chromatin sample and $25 \mu \mathrm{L}$ of protein $\mathrm{G}$ magnetic beads in a total volume of $100 \mu \mathrm{L}$ overnight at $4^{\circ} \mathrm{C}$ on a tube rotator. The beads were then washed and eluted, and the eluted sample was reverse-cross-linked and digested with proteinase $\mathrm{K}$. The eluted DNA was purified using the ReliaPrep FFPE gDNA minipreparation system (Promega) for real-time qPCR. The fold enrichment was calculated as the proportional change from the naïve IgG control without glucose treatment. The antibodies used were naïve rabbit IgG (EMD Millipore, 12-370) and sc14705 (Santa Cruz Biotechnology), sc-133397 (Santa Cruz Biotechnology), 13-2500 (clone 9E10, Thermo Fisher Scientific), and 06-598 (EMD Millipore) for MLX, MLXIP, MYC, and H4ac, respectively. Note that the anti-MLX and anti-MLXIP antibodies used here for ChIP-qPCR experiments appear to have low affinity because, in ChIP-seq (ChIP combined with deep sequencing) experiments, peaks could be called only at the Txnip promoter but nowhere else in the genome. Oligonucleotides used for ChIP-qPCR experiments are listed in Supplemental Table S4, and we confirmed that enrichment was not present at a control locus distal from the promoter (Txnip flanking).

\section{ChIP-seq and data analysis}

For ChIP-seq, libraries were prepared from $10 \mathrm{ng}$ of DNA using the NEB Next ChIP-seq library preparation reagent set for Illumina with NEB Next high-fidelity $2 \times$ PCR master mix according to the manufacturer's instructions (New England Biolabs) with the following modifications: A second 1:1 Ampure cleanup was added after adaptor ligation, the Ampure size selection step prior to PCR was eliminated, and the $72^{\circ} \mathrm{C}$ extension step of the PCR was lengthened to $45 \mathrm{sec}$. Completed libraries were analyzed for insert size distribution on a 2100 BioAnalyzer high-sensitivity kit (Agilent Technologies) or Caliper LabChip GX DNA high-sensitivity reagent kit (PerkinElmer). Libraries were quantified using the Quant-iT PicoGreen dsDNA assay (Life Technologies) and Kapa library quantification kit (Kapa Biosystems) or low-pass sequenc- ing on a MiSeq nano kit (Illumina). Fifty-cycle single-end sequencing was performed on an Illumina HiSeq 2500 for DNA obtained from MLX-WT and MLX-DN samples immunoprecipitated for $\mathrm{H} 4 \mathrm{ac}$ with three replicates for each sample, including input chromatin. For all of these ChIP-seq data, we used BWA /version 0.5.9-r26-dev, default parameter) to align the reads to mouse genome mm9 (MGSCv37 from Sanger) and then marked duplicated reads with Picard (version 1.65 [1160]), with only nonduplicated reads kept by Samtools (parameter "-q 1 -F 1024" version 0.1.18 [r982:295]). To control the quality of the data and estimate the fragment size, the nonduplicated version of SPP (version 1.11) was used to draw cross-correlation with support of $\mathrm{R}$ (version 2.14.0) using the packages caTools (version 1.17) and bitops (version 1.0-6). All of our data passed quality control following ENCODE criterion. Upon manually inspecting the cross-correlation plot generated by SPP, the best fragment size estimates (the smallest fragment size estimated by SPP in all of our cases) were used to extend each read and combine replicates to generate bigwig files to view on IGV (version 2.3.40). After we observed the high consistency between replicates, we scaled the bigwig files and normalized them to $60 \mathrm{M}$ reads so that the heights of peaks between samples are roughly comparable. We generated the normalized H4ac signal on the bigwig files by bigWigSummary from University of California at Santa Cruz and averaged it across gene sets (Kent et al. 2010). In order to identify regions of variable H4 acetylation with MLX genotypes, we considered the read count ratio versus input normalized by total reads; i.e., (Rep1 + Rep2 + Rep3 + 1)/(Input $1+$ Input $2+$ Input $3+1) /($ TotalRep $1+$ TotalRep 2 + TotalRep3 $)^{*}($ TotalInput 1 + TotalInput 2 + TotalInput 3$)$ for $4-\mathrm{kb}$ regions centered around the TSS from the RefSeq database. This allowed for identification of differentially acetylated promoter regions that was further confirmed by ChIP-qPCR. ChIP-seq results are available at the Gene Expression Omnibus (GEO) with GEO accession number GSE74678.

\section{Computational prediction of CHORE motifs}

To predict potential CHORE-like sequences in proximity to $\mathrm{H} 4 \mathrm{ac}$ peaks, we used putative CHORE sequences previously identified from ChIP-seq of MLXIPL (CHREBP) in human adipose and liver tissue (Poungvarin et al. 2015) to generate a position weight matrix. This matrix was used to scan the motif matches on the mouse mm9 genome by STORM (Schones et al. 2007), with a $P$-value cutoff of $1 \times 10^{-5}$.

\section{Real-time $q P C R$}

RNA was extracted from cultured cells with Trizol reagent (Life Technologies) followed by RNEasy minikit (Qiagen) cleanup and DNase treatment. RNA was quantitated, and $500 \mathrm{ng}$ was reverse-transcribed into cDNA using iScript cDNA synthesis kit (Bio-Rad). The resulting cDNA was used for qPCR with SYBR Green (Bio-Rad) with validated oligonucleotides for specific transcripts. Normalized expression of qPCR data was obtained by following the $\Delta \Delta \mathrm{C}_{\mathrm{t}}$ method and using the housekeeper genes Ppia (for cell culture samples) and Hprt (for regeneration studies), which were previously found to be reliable normalization control genes in myoblast culture conditions and muscle regeneration experiments (Hunt et al. 2013). Oligonucleotide sequences used for qPCR are listed in Supplemental Table S4.

\section{RNA-seq and data analysis}

RNA was extracted as described above. Three biological replicates were prepared for RNA-seq with the TruSeq stranded 
mRNA library preparation kit (Illumina) and sequenced on the Illumina HiSeq 2000 platform. FastQ sequences were mapped to the mouse mm9 genome by StrongARM, developed for the Pediatric Cancer Genome Project (Downing et al. 2012). Mapped reads were counted with HTSeq (Anders et al. 2015), and gene-level FPKM (fragments per kilobase per million mapped fragments) values were computed. All sample data were collated into a matrix using $\mathrm{R}(3.0 .1)$ and log start-transformed $\left[\log _{2}(\mathrm{FPKM}+1]\right.$ in STATA/MP 11.2. Genes were statistically tested by class ( $t$-test, unequal variance $t$-test per gene). Bonferroni correction and false discovery rates (FDRs) were determined to allow both strict and modest multiple comparison filtering (Partek Genomics suite 6.6). Six-hundred-fifty-five genes were selected that had an absolute value log ratio of at least 1 in at least one comparison and passed FDR at $5 \%$ (vs. respective controls). We then filtered for genes that showed concordant regulation with MLX-WT, MLX-DN, and MLX shRNA; i.e., genes that change in the same directions in MLX-DN and MLX shRNA samples and do not have the same directional change in the MLX-WT samples. The 80 genes concordantly regulated by MLX were identified because they satisfied a minimum of two out of three of the following requirements: a significant change of at least 1.5-fold compared with control for (1) MLX-WT, (2) MLX-DN, and (3) MLX shRNA; that is, the 80 selected genes were either regulated consistently by both MLX-DN and MLX shRNA or in an opposite fashion by MLX-WT and MLX-DN and/or MLX shRNA. These were analyzed for enriched gene sets by using the SwissProt PIR (SP_PIR) and DAVID. The results of RNA-seq mapping and quantitation, the 80 selected genes, and the enriched gene clusters are presented in Supplemental Table S1. RNA-seq results are available at the Gene Expression Omnibus with accession number GSE70028.

\section{Metabolic analyses}

Extracellular lactate from cell culture medium was quantitated with the glycolysis cell-based assay kit (Cayman Chemical). Glucose uptake was measured with the glucose uptake cell-based assay kit (Cayman Chemical) following the plate-reading method indicated in the manufacturer's instructions. Seahorse analysis was done using a Seahorse XF24 analyzer, the XF glycolysis stress test kit, and the XF cell mito stress test kit after optimization of drug concentrations for $\mathrm{C} 2 \mathrm{C} 12$ cells (the concentrations used are indicated in Fig. 2).

\section{Statistical analyses and calculations}

Data shown represents the mean \pm SEM with a minimum of three biological replicates. Statistical comparisons between groups were done using GraphPad Prism software and one-way or twoway ANOVA with Dunnet's multiple comparison tests for assays with one and two variables, respectively. $Z$-score calculations, RNA-seq graphs, and heat maps were generated with Spotfire DecisionSite software.

\section{Acknowledgments}

We thank Mark Hatley for the anti-myogenin antibody, Sandra Milasta and Doug Green for assistance with Seahorse analysis, Jamy Peng and members of her laboratory for assistance with ChIP experiments, and Scott Olsen, Geoff Neale, and the Genomics facility at the St. Jude Children's Research Hospital Hartwell Center for Bioinformatics and Biotechnology. This work was supported by funding to F.D. from the American Lebanese
Syrian Associated Charities, the Ellison Medical Foundation New Scholar in Aging award, the Glenn Foundation for Medical Research, and the American Federation for Aging Research. R.N.E. was funded by National Institutes of Health/National Cancer Institute grant RO1 CA57138, and P.A.C. was supported by Chromosome Metabolism and Cancer Training Grant T32 CA009657.

\section{References}

Alzhanov DT, McInerney SF, Rotwein P. 2010. Long range interactions regulate Igf2 gene transcription during skeletal muscle differentiation. J Biol Chem 285: 38969-38977.

Anders S, Pyl PT, Huber W. 2015. HTSeq-a Python framework to work with high-throughput sequencing data. Bioinformatics 31: 166-169.

Billin AN, Eilers AL, Queva C, Ayer DE. 1999. Mlx, a novel Maxlike BHLHZip protein that interacts with the Max network of transcription factors. J Biol Chem 274: 36344-36350.

Billin AN, Eilers AL, Coulter KL, Logan JS, Ayer DE. 2000. MondoA, a novel basic helix-loop-helix-leucine zipper transcriptional activator that constitutes a positive branch of a maxlike network. Mol Cell Biol 20: 8845-8854.

Boström P, Wu J, Jedrychowski MP, Korde A, Ye L, Lo JC, Rasbach KA, Boström EA, Choi JH, Long IZ. 2012. A PGC1-a-dependent myokine that drives brown-fat-like development of white fat and thermogenesis. Nature 481: 463-468.

Broholm C, Laye MJ, Brandt C, Vadalasetty R, Pilegaard H, Pedersen BK, Scheele C. 2011. LIF is a contraction-induced myokine stimulating human myocyte proliferation. J Appl Physiol 111: 251-259.

Carroll PA, Diolaiti D, McFerrin L, Gu H, Djukovic D, Du J, Cheng PF, Anderson S, Ulrich M, Hurley JB. 2015. Deregulated Myc requires MondoA/Mlx for metabolic reprogramming and tumorigenesis. Cancer Cell 27: 271-285.

Cha-Molstad H, Saxena G, Chen J, Shalev A. 2009. Glucose-stimulated expression of Txnip is mediated by carbohydrate response element-binding protein, p300, and histone $\mathrm{H} 4$ acetylation in pancreatic $\beta$ cells. $I$ Biol Chem 284: 16898-16905.

Davies MN, O'Callaghan BL, Towle HC. 2008. Glucose activates ChREBP by increasing its rate of nuclear entry and relieving repression of its transcriptional activity. I Biol Chem 283: 24029-24038.

Demontis F, Piccirillo R, Goldberg AL, Perrimon N. 2013a. The influence of skeletal muscle on systemic aging and lifespan. Aging Cell 12: 943-949.

Demontis F, Piccirillo R, Goldberg AL, Perrimon N. 2013b. Mechanisms of skeletal muscle aging: insights from Drosophila and mammalian models. Dis Model Mech 6: 13391352.

Demontis F, Patel VK, Swindell WR, Perrimon N. 2014. Intertissue control of the nucleolus via a myokine-dependent longevity pathway. Cell Rep 7: 1481-1494.

Diolaiti D, McFerrin L, Carroll PA, Eisenman RN. 2015. Functional interactions among members of the MAX and MLX transcriptional network during oncogenesis. Biochim Biophys Acta 1849: 484-500.

Downing JR, Wilson RK, Zhang J, Mardis ER, Pui C-H, Ding L, Ley TJ, Evans WE. 2012. The pediatric cancer genome project. Nat Genet 44: 619-622.

Duputié A, Antoine E, Aptel N, Milligan L, Carbonell F, LelayTaha M-N, Piette J, Weber M, Montarras D, Pinset C. 2012. H19 antisense RNA can up-regulate Igf2 transcription by 
activation of a novel promoter in mouse myoblasts. PLoS One 7: e37923.

Erbay E, Park I-H, Nuzzi PD, Schoenherr CJ, Chen J. 2003. IGF-II transcription in skeletal myogenesis is controlled by mTOR and nutrients. I Cell Biol 163: 931-936.

Florini J, Magri K, Ewton D, James P, Grindstaff K, Rotwein P. 1991a. 'Spontaneous' differentiation of skeletal myoblasts is dependent upon autocrine secretion of insulin-like growth factor-II. J Biol Chem 266: 15917-15923.

Florini JR, Ewton DZ, Magri KA. 1991b. Hormones, growth factors, and myogenic differentiation. Annu Rev Physiol 53: 201-216.

Fulco M, Cen Y, Zhao P, Hoffman EP, McBurney MW, Sauve AA, Sartorelli V. 2008. Glucose restriction inhibits skeletal myoblast differentiation by activating SIRT1 through AMPK-mediated regulation of Nampt. Dev Cell 14: 661-673.

Ge Y, Wu A-L, Warnes C, Liu J, Zhang C, Kawasome H, Terada N, Boppart MD, Schoenherr CJ, Chen J. 2009. mTOR regulates skeletal muscle regeneration in vivo through kinase-dependent and kinase-independent mechanisms. Am I Physiol Cell Physiol 297: C1434-C1444.

Guinez C, Filhoulaud G, Rayah-Benhamed F, Marmier S, Dubuquoy C, Dentin R, Moldes M, Burnol A-F, Yang X, Lefebvre $\mathrm{T}$, et al. 2011. O-GlcNAcylation increases ChREBP protein content and transcriptional activity in the liver. Diabetes 60: 1399-1413.

Hunt L, Tudor E, White J. 2010. Leukemia inhibitory factor-dependent increase in myoblast cell number is associated with phosphotidylinositol 3-kinase-mediated inhibition of apoptosis and not mitosis. Exp Cell Res 316: 1002-1009.

Hunt LC, Upadhyay A, Jazayeri JA, Tudor EM, White JD. 2011. Caspase-3, myogenic transcription factors and cell cycle inhibitors are regulated by leukemia inhibitory factor to mediate inhibition of myogenic differentiation. Skelet Muscle 1: 17.

Hunt L, Gorman C, Kintakas C, McCulloch D, Mackie E, White J. 2013. Hyaluronan synthesis and myogenesis: a requirement for hyaluronan synthesis during myogenic differentiation independent of pericellular matrix formation. I Biol Chem 288: 13006-13021.

Iizuka K, Takeda J, Horikawa Y. 2009. Hepatic overexpression of dominant negative Mlx improves metabolic profile in diabetes-prone C57BL/6J mice. Biochem Biophys Res Commun 379: 499-504.

Ikemoto-Uezumi M, Uezumi A, Tsuchida K, Fukada S-i, Yamamoto H, Yamamoto N, Shiomi K, Hashimoto N. 2015. Pro-insulin-like growth factor-II ameliorates age-related inefficient regenerative response by orchestrating self-reinforcement mechanism of muscle regeneration. Stem Cells 33: 24562468.

Iruela-Arispe ML, Liska DJ, Sage EH, Bornstein P. 1993. Differential expression of thrombospondin 1, 2, and 3 during murine development. Dev Dyn 197: 40-56.

Jiang B-H, Aoki M, Zheng JZ, Li J, Vogt PK. 1999. Myogenic signaling of phosphatidylinositol 3-kinase requires the serinethreonine kinase Akt/protein kinase B. Proc Natl Acad Sci 96: 2077-2081.

Kaadige MR, Yang J, Wilde BR, Ayer DE. 2015. MondoA-Mlx transcriptional activity is limited by $\mathrm{mTOR}-$ MondoA interaction. Mol Cell Biol 35: 101-110.

Kawaguchi T, Osatomi K, Yamashita H, Kabashima T, Uyeda K. 2002. Mechanism for fatty acid 'sparing' effect on glucose-induced transcription regulation of carbohydrate-responsive element-binding protein by AMP-activated protein kinase. $I$ Biol Chem 277: 3829-3835.
Kent WJ, Zweig AS, Barber G, Hinrichs AS, Karolchik D. 2010. BigWig and BigBed: enabling browsing of large distributed datasets. Bioinformatics 26: 2204-2207.

Kou K, Rotwein P. 1993. Transcriptional activation of the insulinlike growth factor-II gene during myoblast differentiation. Mol Endocrinol 7: 291-302.

Lee JV, Carrer A, Shah S, Snyder NW, Wei S, Venneti S, Worth AJ, Yuan Z-F, Lim H-W, Liu S. 2014. Akt-dependent metabolic reprogramming regulates tumor cell histone acetylation. Cell Metab 20: 306-319.

Levinovitz A, Jennische E, Oldfors A, Edwall D, Norstedt G. 1992. Activation of insulin-like growth factor II expression during skeletal muscle regeneration in the rat: correlation with myotube formation. Mol Endocrinol 6: 1227-1234.

Ma L, Tsatsos NG, Towle HC. 2005. Direct role of ChREBP.Mlx in regulating hepatic glucose-responsive genes. I Biol Chem 280: 12019-12027.

Ma L, Robinson LN, Towle HC. 2006. ChREBP.Mlx is the principal mediator of glucose-induced gene expression in the liver. $I$ Biol Chem 281: 28721-28730.

Markljung E, Jiang L, Jaffe JD, Mikkelsen TS, Wallerman O, Larhammar M, Zhang X, Wang L, Saenz-Vash V, Gnirke A. 2009. ZBED6, a novel transcription factor derived from a domesticated DNA transposon regulates IGF2 expression and muscle growth. PLoS Biol 7: e1000256.

McFerrin LG, Atchley WR. 2012. A novel N-terminal domain may dictate the glucose response of Mondo proteins. PLoS One 7: e34803.

Minako I, Benny H-JC, Motoyuki K, Ming L, Byounghoon H, Heinrich T, Robert AH, Lawrence C. 2014. MondoA deficiency enhances sprint performance in mice. Biochem J 464: 35-48.

Minn AH, Hafele C, Shalev A. 2005. Thioredoxin-interacting protein is stimulated by glucose through a carbohydrate response element and induces $\beta$-cell apoptosis. Endocrinology 146: 2397-2405.

Nam SO, Yotsumoto F, Miyata K, Fukagawa S, Yamada H, Kuroki M, Miyamoto S. 2015. Warburg effect regulated by amphiregulin in the development of colorectal cancer. Cancer Med 4: $575-587$.

Nedachi T, Kadotani A, Ariga M, Katagiri H, Kanzaki M. 2008. Ambient glucose levels qualify the potency of insulin myogenic actions by regulating SIRT1 and FoxO3a in C2C12 myocytes. Am I Physiol Endocrinol Metab 294: E668-E678.

Parikh H, Carlsson E, Chutkow WA, Johansson LE, Storgaard H, Poulsen P, Saxena R, Ladd C, Schulze PC, Mazzini MJ. 2007. TXNIP regulates peripheral glucose metabolism in humans. PLoS Med 4: e158.

Pedersen BK, Febbraio MA. 2012. Muscles, exercise and obesity: skeletal muscle as a secretory organ. Nat Rev Endocrinol 8: 457-465.

Peterson CW, Stoltzman CA, Sighinolfi MP, Han K-S, Ayer DE. 2010. Glucose controls nuclear accumulation, promoter binding, and transcriptional activity of the MondoA-Mlx heterodimer. Mol Cell Biol 30: 2887-2895.

Piccirillo R, Demontis F, Perrimon N, Goldberg AL. 2014. Mechanisms of muscle growth and atrophy in mammals and Drosophila. Dev Dyn 243: 201-215.

Postic C, Dentin R, Denechaud P-D, Girard J. 2007. ChREBP, a transcriptional regulator of glucose and lipid metabolism. Annu Rev Nutr 27: 179-192.

Poungvarin N, Chang B, Imamura M, Chen J, Moolsuwan K, SaeLee C, Li W, Chan L. 2015. Genome-wide analysis of ChREBP binding sites on male mouse liver and white adipose chromatin. Endocrinology 156: 1982-1994. 
Rai M, Demontis F. 2015. Systemic nutrient and stress signaling via myokines and myometabolites. Annu Rev Physiol doi: 10.1146/annurev-physiol-021115-105305.

Sakamoto K, Yamaguchi S, Ando R, Miyawaki A, Kabasawa Y, Takagi M, Li CL, Perbal B, Katsube K-i. 2002. The nephroblastoma overexpressed gene $(\mathrm{NOV} / \mathrm{ccn} 3)$ protein associates with Notch1 extracellular domain and inhibits myoblast differentiation via Notch signaling pathway. I Biol Chem 277: 29399-29405.

Schneider CA, Rasband WS, Eliceiri KW. 2012. NIH Image to ImageJ: 25 years of image analysis. Nat Methods 9: 671-675.

Schones DE, Smith AD, Zhang MQ. 2007. Statistical significance of cis-regulatory modules. BMC Bioinformatics 8: 19 .

Shen L, O'Shea JM, Kaadige MR, Cunha S, Wilde BR, Cohen AL, Welm AL, Ayer DE. 2015. Metabolic reprogramming in triplenegative breast cancer through Myc suppression of TXNIP. Proc Natl Acad Sci 112: 5425-5430.

Stoltzman CA, Peterson CW, Breen KT, Muoio DM, Billin AN, Ayer DE. 2008. Glucose sensing by MondoA:Mlx complexes: a role for hexokinases and direct regulation of thioredoxin-interacting protein expression. Proc Natl Acad Sci 105: 6912-6917.

Stoltzman CA, Kaadige MR, Peterson CW, Ayer DE. 2011. MondoA senses non-glucose sugars: regulation of thioredoxin-interacting protein (TXNIP) and the hexose transport curb. I Biol Chem 286: 38027-38034.
Tixier V, Bataillé L, Etard C, Jagla T, Weger M, DaPonte JP, Strähle U, Dickmeis T, Jagla K. 2013. Glycolysis supports embryonic muscle growth by promoting myoblast fusion. Proc Natl Acad Sci 110: 18982-18987.

Webster KA, Gunning P, Hardeman E, Wallace DC, Kedes L. 1990. Coordinate reciprocal trends in glycolytic and mitochondrial transcript accumulations during the in vitro differentiation of human myoblasts. J Cell Physiol 142: 566-573.

Wu N, Zheng B, Shaywitz A, Dagon Y, Tower C, Bellinger G, Shen C-H, Wen J, Asara J, McGraw TE. 2013. AMPKdependent degradation of TXNIP upon energy stress leads to enhanced glucose uptake via GLUT1. Mol Cell 49: 1167-1175.

Yahiaoui L, Gvozdic D, Danialou G, Mack M, Petrof BJ. 2008. CC family chemokines directly regulate myoblast responses to skeletal muscle injury. J Physiol 586: 3991-4004.

Yamashita H, Takenoshita M, Sakurai M, Bruick RK, Henzel WJ, Shillinglaw W, Arnot D, Uyeda K. 2001. A glucose-responsive transcription factor that regulates carbohydrate metabolism in the liver. Proc Natl Acad Sci 98: 9116-9121.

Yoon M-S, Chen J. 2008. PLD regulates myoblast differentiation through the mTOR-IGF2 pathway. I Cell Sci 121: 282-289.

Yu F-X, Goh S-R, Dai R-P, Luo Y. 2009. Adenosine-containing molecules amplify glucose signaling and enhance txnip expression. Mol Endocrinol 23: 932-942. 


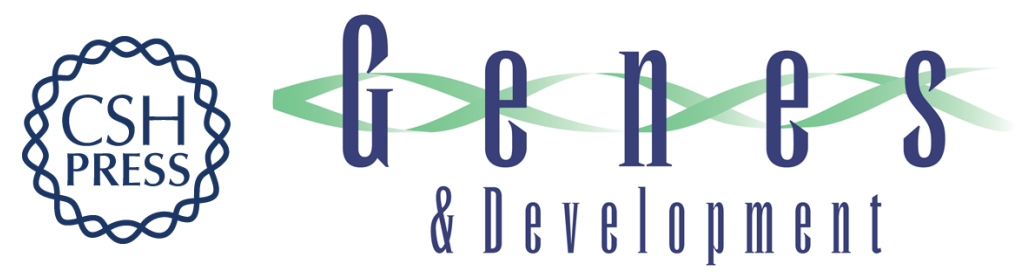

\section{The glucose-sensing transcription factor MLX promotes myogenesis via myokine signaling}

Liam C. Hunt, Beisi Xu, David Finkelstein, et al.

Genes Dev. 2015, 29: originally published online November 19, 2015

Access the most recent version at doi:10.1101/gad.267419.115

\section{Supplemental http://genesdev.cshlp.org/content/suppl/2015/11/18/gad.267419.115.DC1 Material}

References This article cites 62 articles, 24 of which can be accessed free at: http://genesdev.cshlp.org/content/29/23/2475.full.html\#ref-list-1

Creative This article is distributed exclusively by Cold Spring Harbor Laboratory Press for the first Commons six months after the full-issue publication date (see

License http://genesdev.cshlp.org/site/misc/terms.xhtml). After six months, it is available under a Creative Commons License (Attribution-NonCommercial 4.0 International), as described at http://creativecommons.org/licenses/by-nc/4.0/.

Email Alerting Receive free email alerts when new articles cite this article - sign up in the box at the top Service right corner of the article or click here.

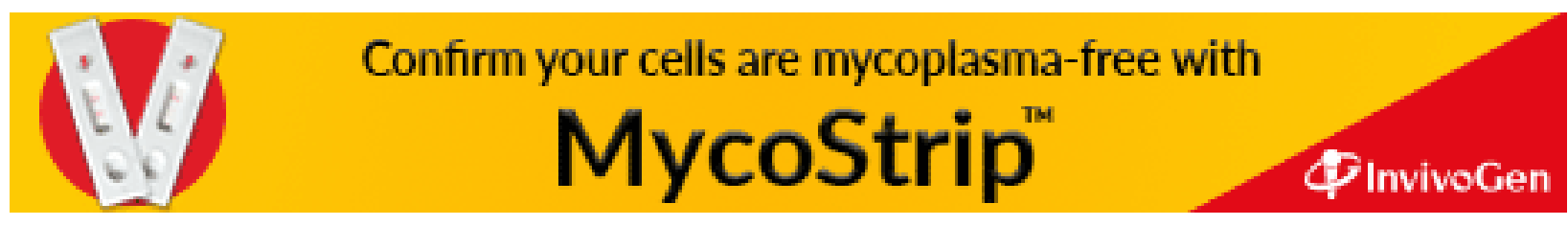

